Portland State University

PDXScholar

\title{
Quasi-Steady Capillarity-Driven Flows in Slender Containers with Interior Edges
}

\author{
Mark M. Weislogel \\ Portland State University, weisloge@pdx.edu \\ J. Alex Baker \\ Portland State University \\ Ryan M. Jenson \\ Portland State University
}

Follow this and additional works at: https://pdxscholar.library.pdx.edu/mengin_fac

Part of the Mechanical Engineering Commons

Let us know how access to this document benefits you.

\section{Citation Details}

Mark M. Weislogel, J. Alex Baker and Ryan M. Jenson (2011). Quasi-steady capillarity-driven flows in slender containers with interior edges. Journal of Fluid Mechanics, 685, pp 271-305 doi:10.1017/ jfm.2011.314

This Article is brought to you for free and open access. It has been accepted for inclusion in Mechanical and Materials Engineering Faculty Publications and Presentations by an authorized administrator of PDXScholar. Please contact us if we can make this document more accessible: pdxscholar@pdx.edu. 


\title{
Quasi-steady capillarity-driven flows in slender containers with interior edges
}

\author{
Mark M. Weislogel $\dagger$, J. Alex Baker and Ryan M. Jenson \\ Department of Mechanical and Materials Engineering, Portland State University, PO Box 751, Portland, \\ OR 97207, USA
}

(Received 21 January 2011; revised 24 June 2011; accepted 19 July 2011; first published online 23 September 2011)

In the absence of significant body forces the passive manipulation of fluid interfacial flows is naturally achieved by control of the specific geometry and wetting properties of the system. Numerous 'microfluidic' systems on Earth and 'macrofluidic' systems aboard spacecraft routinely exploit such methods and the term 'capillary fluidics' is used to describe both length-scale limits. In this work a collection of analytic solutions is offered for passive and weakly forced flows where a bulk capillary liquid is slowly drained or supplied by a faster capillary flow along at least one interior edge of the container. The solutions are enabled by an assumed known pressure (or known height) dynamical boundary condition. Following a series of assumptions this boundary condition can be in part determined a priori from the container dimensions and further quantitative experimental evidence, but not proof, is provided in support of its expanded use herein. In general, a small parameter arises in the scaling of the problems permitting a decoupling of the edge flow from the global bulk meniscus flow. The quasi-steady asymptotic system of equations that results may then be easily solved in closed form for a useful variety of geometries including uniform and tapered sections possessing at least one critically wetted interior edge. Draining, filling, bubble displacement and other imbibing flows are studied. Cursory terrestrial and drop tower experiments agree well with the solutions. The solutions are valued for the facility they provide in computing designs for selected capillary fluidics problems by way of passive transport rates and meniscus displacement. Because geometric permutations of any given design are myriad, such analytic tools are capable of efficiently identifying and comparing critical design criteria (i.e. shape and size) and the impact of various wetting conditions resulting from the fluid properties and surface conditions. Sample optimizations are performed to demonstrate the utility of the method.

Key words: capillary flows, lubrication theory, microfluidics

\section{Introduction and scope}

Engineered microfluidic processes on Earth such as coolant circulation in capillarydriven heat pipes studied by Suman \& Kumar (2005), Sobhan, Rag \& Peterson (2007) and Zhang, Watson \& Wong (2007) demonstrate the degree to which passive control may be maintained provided that the characteristic system dimension is small, i.e. capillary length $\lesssim O(1 \mathrm{~mm})$. In the near weightless environment aboard spacecraft, the

$\dagger$ Email address for correspondence: mmw@cecs.pdx.edu 

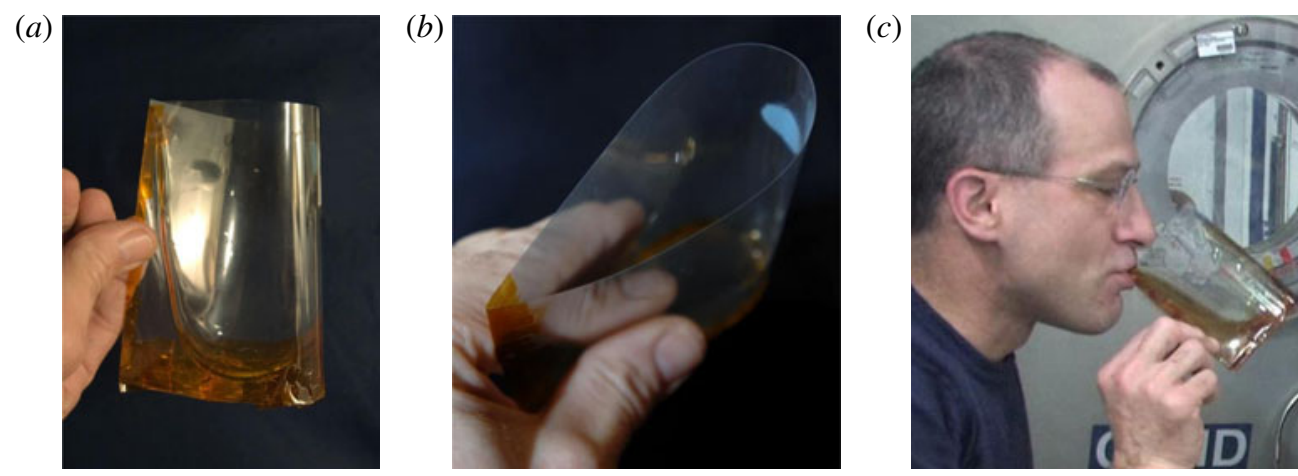

FIGURE 1. 'Low-g coffee cup': (a) single edge wetting, $(b)$ section view, $(c)$ astronaut Donald Pettit drinking from cup aboard the International Space Station. The drinking process is essentially a capillarity-limited draining problem analysed herein.

reduced body force permits identical control, but for systems with significantly larger characteristic dimensions, i.e. capillary length $\lesssim O(1 \mathrm{~m})$. The latter processes might be called 'macrofluidic,' and dramatically expand opportunities to exploit capillary forces to carry out tasks in non-Earthly ways, such as the storage and management of liquid fuels or cryogens by the tonne for spaceflight as addressed by Chato (2008), or the circulation and separation of large quantities of water in spacecraft life support systems as addressed by Weislogel, Thomas \& Graf (2009b). The term 'capillary fluidics' is adopted to describe both length-scale limits and is loosely defined as the management of fluid interfacial systems by relying strongly on the passive means afforded by system geometry and wetting conditions.

One particularly useful geometric construct in capillary fluidic systems is the interior edge (or interior corner, wedge, groove, etc.). For example, employed in a polygonal container or conduit, if the interior edge included angle and liquid-fluid contact angle are small enough, the edge is spontaneously filled by the wetting fluid. This critical edge wetting condition provides an attractive and effective means for truly passive fluids transport and control. On the macro-scale, for a variety of applications it can be used to drain large fluid quantities from one region of a container to another (i.e. Weislogel \& Collicott 2004; Chato \& Martin 2006), or to passively separate immiscible fluid phases for further processing. An illustrative example of the former is shown in figure 1 where astronaut Donald Pettit drinks from the edge of a 'low- $g$ coffee cup' in the nearly weightless environment of the International Space Station (ISS) (during spaceflight STS-126, November 2008, search internet under 'Astronaut Coffee Cup'). The static interface profile is shown in figure 1(a). The cross-section perspective of the cup is shown in figure $1(b)$, where the capillarity-driven edge flow along the single interior edge acts to drain the contents as liquid is removed at the lip of the cup, figure 1(c). In effect, the capillary edge flow replaces gravity in the role of managing the liquid position and the astronaut drinks almost as if on Earth.

A perhaps more applied example of passive edge flows is provided in figure 2, where a capillarity-driven migration along the long interior edges of a tapered rectangular slot is shown for a bubbly flow also during experiments performed aboard the ISS by astronaut Sunita Williams (Weislogel et al. 2009a). Across the container the edge flows displace the gas volume by collecting the liquid at the vertex. Within the corners themselves, however, the edge flows work to concentrate the entrained 


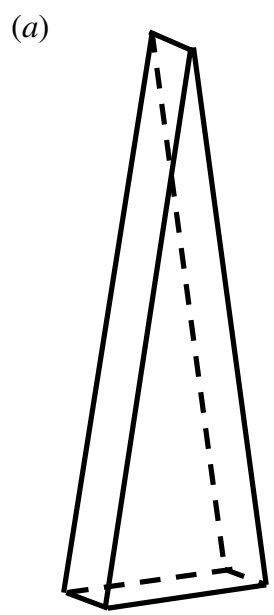

Bubbly liquid

$(b)$

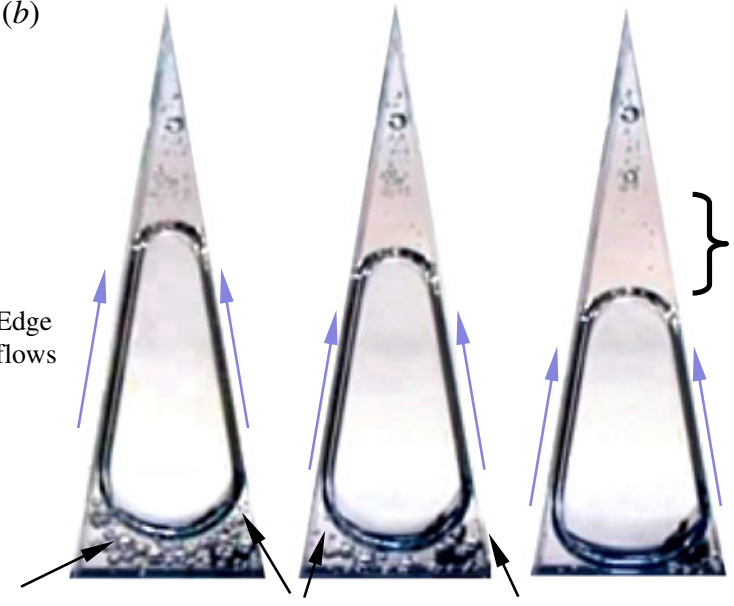

Forced coalescence region

FIGURE 2. (Colour online available at journals.cambridge.org/flm) Passive migration and separation of a bubbly flow in a microgravity environment (Interior Corner Flow Experiment aboard ISS, Weislogel et al. 2009a). An 8.95 tapered rectangular slot $127 \mathrm{~mm}$ tall and $10 \mathrm{~mm} \times 40 \mathrm{~mm}$ at the base is partially filled with $9.02 \mathrm{ml}$ of $2 \mathrm{cs}$ PDMS: $(a)$ a wire model of the test container, $(b)$ a sequence of images at approximately $1 \mathrm{~min}$ intervals following injection of a bubbly flow into the base of the container. The edge flows identified by arrows pass nearly bubble-free as indicated by the braced region at far right.

bubbles in the liquid. These bubbles are forced against the interface along the edges where the vast majority of them coalesce. The geometry provides both reorientation and separation operations passively. Simple analytical expressions to predict such flows in general are the pursuit of this work.

In this paper, a brief review of capillary edge flow is provided along with a thread of published works and elaboration leading to the postulation of a dynamical boundary condition upon which all of the subsequent analytical solutions depend. The boundary condition is only employed for slender geometries, either of uniform or tapering section, and possessing sharp interior edges. Experimental evidence, but not proof, is provided in support of its use. Lists of local and global flow assumptions are then presented that reduce the general system to typically $m+3$ equations, where $m$ is the number of critically wetted interior edges for the container: $m$ edge flow mass-balance equations, two bulk meniscus mass balance equations, and one volume constraint. The equations are non-dimensionalized by demonstrated scales giving rise to two fundamental geometric parameters that weigh the influence of $j$ th edge saturation $\beta_{j}$ and edge flow uniformity $\omega_{j}$. The system is dramatically simplified in the limit of low saturation $\left(\beta_{j} \ll 1\right)$ in containers with $m$ regular wetted edges $\left(\omega_{j}=1\right)$. The latter constraint is not necessary for solutions, but is often employed for clarity in this presentation. Sample solutions are then outlined to illustrate the general method, beginning with exact solutions for simple draining and bubble displacement flows. Cursory terrestrial and drop tower experiments are provided that corroborate the analytical predictions. With this encouragement, the dimensional governing equations are re-formulated at $O(1)$ and solved for passive and weakly forced flows in a number of practical geometries. The new closed-form solutions demonstrate the quick allanalytic method for determining designs for such capillary fluidic systems prior to the 
more involved task of full numerical computations. Suggestions for continued research are highlighted in the summary.

\section{Review of capillarity-driven flows along interior edges}

\subsection{Edge wetting condition}

For sharp interior edges formed by planar walls, spontaneous flows occur when the critical geometric wetting condition $\theta<\pi / 2-\alpha$ is satisfied, where $\theta$ is the equilibrium contact angle of the wetting fluid and $\alpha$ is half the included angle of the planar walls. The mathematical grounds on which this and other critical geometric wetting phenomena are based are credited to mathematicians Concus \& Finn (1969) who demonstrated a discontinuous behaviour of the fluid surface as the 'critical value' is crossed, and established, through proofs of non-existence, that no other non-edgefilling surfaces are possible. Earlier heuristic descriptions of critical wetting conditions have appeared in the literature, see Bankoff (1957), Griffith \& Wallis (1960) and Kast (1964) cited in Collier (1972) (equation (4.18)) with perhaps the most notable and least well-known mathematical contribution being that of Chernous'ko (1968).

Because such critical wetting phenomena may also depend in a complex manner on other geometric quantities such as overall container size, shape, fill fraction, and wetting conditions, the discontinuous, or even nearly discontinuous, geometric wetting condition for a particular system is referred to in general as the 'Concus-Finn condition' for the given geometry, see Collicott \& Weislogel (2004), Akhtara et al. (2009), and Wang et al. (2010). Under this definition, although only one may occur at a time, more than one Concus-Finn condition is possible for a single container - three are demonstrated by Tavan (2009). For partially wetting systems with finite contact angle hysteresis, at least the advancing contact angle should be used when evaluating Concus-Finn wetting conditions in the short term. Conversely, at most, the receding contact angle should be used when evaluating Concus-Finn de-wetting conditions. However, thermal and mechanical perturbations over time appear to bring about the mathematically idealized conditions based on the equilibrium contact angle alone; see Concus, Finn \& Weislogel (2000). Lastly, it is of interest to note that no augmentation of the Concus-Finn condition for planar walls $(\theta<\pi / 2-\alpha)$ has been reported despite the wealth of recent microfluidics research (i.e. Van der Waals and Lennard-Jones fluids where the idealized three-phase contact line model breaks down, Yi \& Wong 2007, or for micro/nano-scale systems where line tension and contact line curvature significantly alter local wetting conditions, Yonemoto \& Kunugi 2008).

\subsection{Simple example of a capillary edge draining flow}

A number of important edge flow characteristics are introduced in parallel with a discussion of a simple capillary edge draining experiment. As depicted in figure 3( $a$ ), a $1 \mathrm{~mm}$ inner diameter, thin-walled rounded-corner square fused quartz tube is sealed at its rightmost end and completely filled with perfectly wetting low-vapourpressure polydimethylsiloxane (tube dimensions: internal face $1 \mathrm{~mm}$, wall thickness $0.15 \mathrm{~mm}$, corner radius $R_{c}=0.124 \mathrm{~mm}$; fluid properties: $5 \mathrm{cs}$ PDMS at $20^{\circ} \mathrm{C}$ : density $\rho=890 \mathrm{~kg} \mathrm{~m}^{-3}$, surface tension $\sigma=0.018 \mathrm{~N} \mathrm{~m}^{-1}$, viscosity $\mu=0.00445 \mathrm{~kg} \mathrm{~m}^{-1} \mathrm{~s}^{-1}$, corner half-angle $\alpha=\pi / 4$, and contact angle $\theta=0^{\circ}$ ). The tube is placed horizontally over a backlight panel mounted on a level translation stage. A roll of absorbent tissue is then brought into contact with the open leftmost end of the tube. The liquid near the tube opening quickly wicks into the smaller pores of the tissue as the counterflow of air may or may not be drawn through the tissue and into the tube. A perspective 
(a)

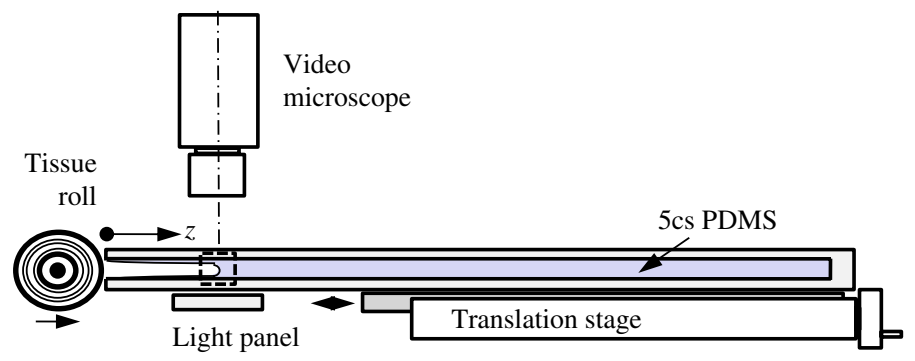

(b)

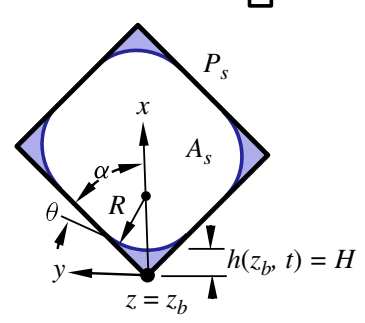

(c)

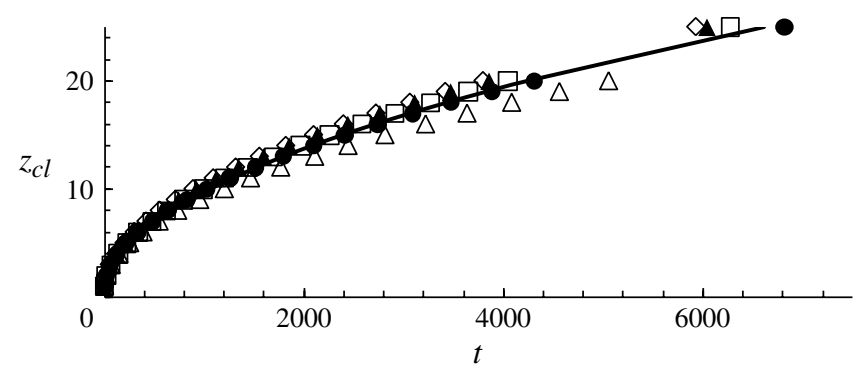

FIgURE 3. (Colour online) (a) Schematic of a thin-walled rounded square tube draining experiment. Flow is initiated once the tissue roll is brought into contact with the leftmost open end of the tube. $(b)$ Perspective of edge draining flow with magnified cross-section at $z=z_{b}$ with relevant notation; i.e. cross-sectional area $A_{s}$, perimeter $P_{s}$, de Lazzer et al. (1996) bulk meniscus radius of curvature $R$, and meniscus height $h\left(z_{b}, t\right)=H$. (c) Plot of five repeat experiments (symbols) for $z_{c l}(\mathrm{~mm})$ versus $t$ (s) with theoretical prediction from (4.41), where $z_{2} \equiv z_{c l}$ (solid line).

view of the flow is shown in figure $3(b)$ with an edge flow section on the right employing notation to be described shortly. The bulk meniscus slowly recedes as the interior edges pump liquid from the bulk to the tissue - in much the same way as the 'astronaut coffee cup' is drained in figure 1. The bulk meniscus region is tracked and recorded by video microscope. For these long duration experiments the impact of tube level and precision, tissue resistance to air ingestion, saturation, particulate contamination, and the effects of temperature-dependent fluid properties including evapouration are all considered and demonstrated to be within the constraints of the pending modelling assumptions.

The interior edge flows are driven by gradients in capillary pressure along the edge and rapidly become slender, figure $3(b)$. This situation permits the increasingly appropriate parallel flow and dominant cross-stream curvature assumptions. The former assumption often negates inertia as a significant force despite the high rates of flow possible in certain large-length-scale capillary fluidic systems. The parallel nature of the edge flow also means that velocities normal to the contact line are small compared to velocities parallel to it, and that the static contact angle provides an increasingly 
(a)

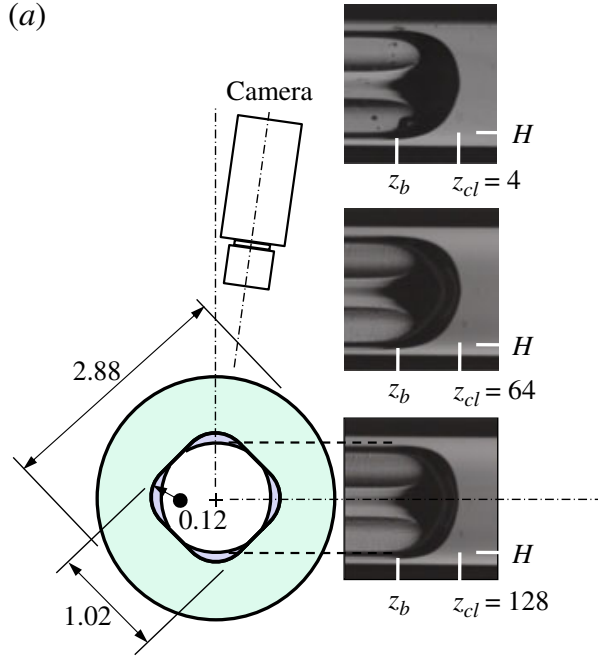

(b)

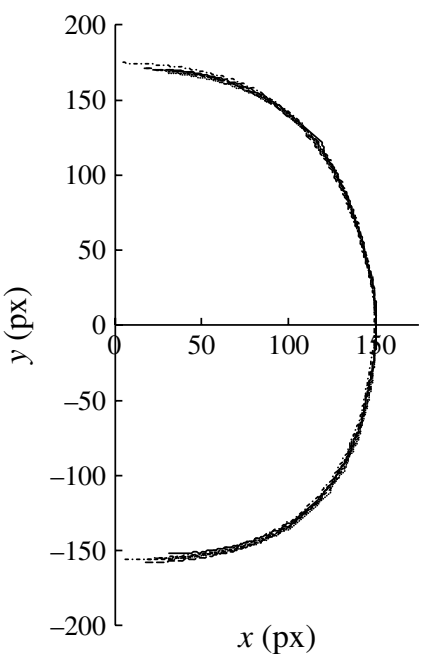

FIGURE 4. (Colour online) Thick-walled rounded-corner square tube draining experiments with 5cs PDMS. (a) Tube section view with sequence of bulk meniscus image profiles during draining, dimensions in $\mathrm{mm}$. Selected centreline $z_{c l}$ locations noted and $z_{b}$ denotes beginning of the bulk meniscus region. Tissue particles are observed to diffuse a short distance upstream (see $z_{c l}=4 \mathrm{~mm}$ ). (b) Overlay of six bulk meniscus interface profiles at different $z$-locations during flow. The $\left(z_{c l}(\mathrm{~mm}), t(\mathrm{~min})\right)$ profiles shown are for: $(4,4.1),(8,15.8),(16,119.7),(32$, $512.2),(64,1936.4)$, and $(128,9148.3)$. Optical distortions are identical for each image and the interfaces are essentially coincident in all cases.

accurate approximation for the free-surface boundary condition in planes perpendicular to the edge axes. Thus, only the $z$-component momentum equation must be solved for the local velocity profiles. (The displaced air is ignored as a passive gas.) The velocity field is integrated over the section area and used in local volume balances to compute second-order nonlinear PDEs for the evolution of the edge flow surface profile $h_{j}(z, t)$ for the $j$ th of $m$ edge flows, $h_{j}(z, t)$ being referred to as the liquid 'height' measured normal to the $z$-axis in the plane bisecting the interior edge. After identification of suitable boundary conditions, solutions to these equations provide each $j$ th edge flow rate.

As identified in figure $3(b)$, the draining bulk meniscus centreline location $z_{c l}$ $\left(\approx z_{b} \equiv z_{2}\right)$ is plotted as a function of time $t$ in figure $3(c)$ for five repeat experiments. The long time behaviour is expected to be $\sim t^{1 / 2}$ and a theoretical curve based on the proposed model assumptions is included on the figure and will be discussed in $\S 4$. To reduce optical distortions for comparisons of bulk interface and edge flow profiles, the experiments are repeated using thick-walled rounded square tubes (drawn quartz tube with $2.88 \mathrm{~mm}$ circular outer diameter, $1.02 \mathrm{~mm}$ rounded square, $R_{c}=0.12 \mathrm{~mm}$, and $200 \mathrm{~mm}$ length: area $A_{s}=1.007 \mathrm{~mm}^{2}$, and perimeter $P_{s}=3.827 \mathrm{~mm}$ ). Selected bulk interface profiles from one such test are overlaid in figure 4 such that bulk menisci centrelines $z_{c l}$ are coincident. Clearly, the bulk meniscus rapidly establishes a constant curvature condition that maintains for the duration of this $152.5 \mathrm{~h}$ experiment. (Note: a consistent asymmetry in the interface images is noticeable and caused by an uncorrected optical distortion amplified by a slight out-of-vertical line of observation as depicted in the exaggerated sketch in figure $4 a$.) 
Because the tissue paper provides a large-volume low-capillary-pressure sink, the boundary condition at the open tube end is effectively a known meniscus height condition for all interior edges where $h(0, t) \approx 0$, referred to as a sink location condition. The question becomes one of what is, and where to apply, a second boundary condition. As discussed in reference to figure 4, as time and distance increase, the essentially constant-curvature bulk meniscus region shrinks to a vertical line as conjectured in Weislogel \& Lichter (1998). The pivotal assumption of this research is that the height $h_{j}(z, t)$ of each interior edge flow corresponds to the constant-height $H_{j}$, or constant-curvature, conditions of de Lazzer et al. (1996) in the vicinity of the bulk meniscus which at long times is compressed to a line. In other words, $z_{b} \approx z_{c l}$ and $h_{j}\left(z_{b}(t)\right) \approx H_{j}$ as identified in figure $3(b)$. It is further argued that for weakly $z$-dependent geometries $h_{j}\left(z_{b}(t)\right) \approx H_{j}(z)$. With such a solution-dependent dynamical boundary condition an important variety of bulk capillary flows governed by interior edge flows may be approximated and easily solved. Lastly, it is important to note for this flow that the saturation level along the edge flow region of the container can be small. Thus, the edge flows advance far and fast while the bulk meniscus recedes a short distance and slowly, suggesting two very different time scales for the flow.

\section{Edge flow review: literature, notation, assumptions, and equations}

For a record of model development for sharp and rounded interior edge flows leading to the present work see Ayyaswamy, Catton \& Edwards (1974), Ransohoff \& Radke (1988), Dong \& Chatzis (1995), Kovscek \& Radke (1996), Romero \& Yost (1996), de Lazzer et al. (1996), Weislogel \& Lichter (1998), and Chen, Weislogel \& Nardin (2006). Extensive experimental verification is reported and numerous other related works could be cited. An assortment of solutions for open channels and conduits are collected by Weislogel (2003), and gravity draining problems are solved by Bico \& Quéré (2002), Ramé \& Weislogel (2009), and Ponomarenko, Clanet \& Quéré (2011). Capillary fluidics systems are demonstrated on Earth by Khare et al. (2007) and in low- $g$ environments by Weislogel et al. (2009b). Efficient numerical techniques have been demonstrated by Klatte et al. (2008) as have methods to account for significant inertia by Rosendahl, Ohlhoff \& Dreyer (2004).

A further simplified draining flow example is discussed here to introduce the notation and method to compute the solution-dependent meniscus height boundary condition $H_{j}$ at a bulk meniscus for the $j$ th of $m$ edge flows. The list of assumptions is then provided for both local edge and bulk meniscus flows. A brief review of the resulting governing edge flow equations is given before introducing the new solutions.

\subsection{Notation and known height boundary condition $H$}

Figure 5 depicts the capillary edge draining flow of figures 3 and 4, but for a right cylinder with constant section where for simplicity only one interior edge is wetted by the liquid - after the example flow in figure 1 , where $j=m=1$. The container section resembles that of an ice cream cone and has been referred to as such in a static analysis by Concus \& Finn (1990). The section on the right consists of two intersecting planar surfaces whose acute faces are tangent to an otherwise right circular cylinder. This particular section geometry will be adopted frequently throughout this presentation for brevity, but will generally be referred to as an $m=1$ section since only one interior edge is wetted. As shown in the section in figure $5(b)$, the interior half-angle at the edge is $\alpha$ and the contact angle for the fluid is $\theta$. The $z$-axis aligns 
(a)

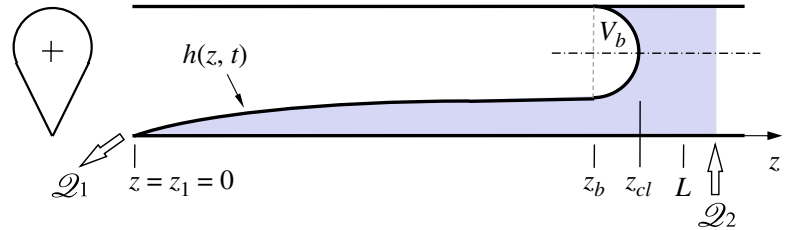

(b)

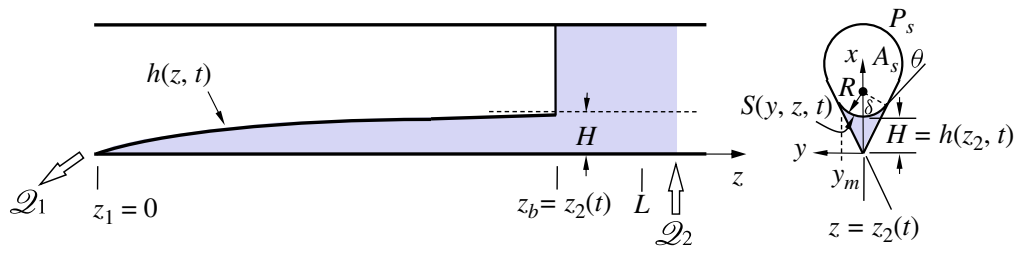

FIGURE 5. (Colour online) An $m=1$ section for draining flows: $(a)$ sketch of actual flow, (b) model interface profile with section at $z=z_{b}=z_{c l}=z_{2}$ with notation used for de Lazzer et al. method to compute $H . \mathscr{Q}_{2}=0$ for unassisted draining problems.

with the container edge and the meniscus height $h(z, t)$ is measured from the edge to the free surface in the $x-z$-plane. As identified on the figure the sink location is stationary at $z \equiv z_{1}=0$, where $h\left(z_{1}\right) \equiv 0$. The bulk meniscus and centreline locations are identified by $z_{b}$ and $z_{c l}$, respectively. The constant height location intersects $h(z, t)$ at the point $h\left(z_{2}(t)\right)=H$. Despite the unbounded nature of this flow, $z_{2} \sim L$ is chosen, where $L$ is an arbitrary length scale. The primary dependent variable is the $z$-component velocity $w(x, y, z, t)$ along the $z$-axis, but the primary velocity gradients are confined to the cross-flow $x-y$-plane; see cross-sections sketched in figures $3(b)$ and $5(b)$.

For a right polygonal cylindrical section of infinite length, de Lazzer et al. (1996) apply the divergence theorem to the Young-Laplace-Gauss equation and, by assuming Concus-Finn edge-wetting configurations, establish a closed-form method to compute the static equilibrium one-dimensional radius of curvature $R$ in such a container for $g=0$. The merits of the method are reviewed by Weislogel \& Collicott (2004) who highlight the limitations identified by Finn \& Neel (1999) before successfully extending the method to families of cylinders with irregular section, symmetric subelements, curved wall elements, planar unconnected vanes, weakly tapered sections, and planar walls of differing contact angles; see also Weislogel (2001) and Weislogel $\&$ Nardin (2005).

Experimental evidence continues to support the broadened application of the de Lazzer et al. method for the flow phenomena of practical interest here; see Weislogel \& Lichter (1998), Weislogel (2001), and Weislogel et al. (2007). The method applied to the $m=1$ section is sketched in figure $5(b)$ with relevant notation, from which the closed-form result is

$$
R=\frac{P_{s} \cos \theta}{2 \Sigma}\left(1-\left(1-\frac{4 \Sigma A_{s}}{P_{s}^{2} \cos ^{2} \theta}\right)^{1 / 2}\right) \equiv f H,
$$

where $P_{s}$ is the perimeter of the container section, $A_{s}$ is the container cross-sectional area, and $\Sigma, F_{A}$, and $f$ are the respective normalized flow area, flow area, and cross-flow interface curvature functions:

$$
\Sigma=\frac{F_{A}}{f^{2}} \quad F_{A}=f^{2}\left(\frac{\cos \theta \sin \delta}{\sin \alpha}-\delta\right), \quad f=\frac{\sin \alpha}{\cos \theta-\sin \alpha},
$$

where $\delta \equiv \pi / 2-\alpha-\theta$. 


\subsection{Quasi-steady edge flows: assumptions}

For the flow idealized in figure $5(b)$, in addition to constant properties and incompressibility, the numerous simplifications discussed in $\S 2$ are formalized and presented here in list form, categorized by their impact at either edge flow or bulk meniscus flow levels.

\subsubsection{Edge flow assumptions}

(a) The edge flow quickly becomes slender, $\epsilon^{2} \ll 1$, where the slenderness parameter $\epsilon \equiv H / L$. In general, this assumption permits application of lubrication theory, largely negating inertia when $\epsilon^{2} S u \sin ^{4} \alpha / f \ll 1\left(S u \equiv \rho \sigma H / \mu^{2}\right)$, streamwise freesurface curvature when $\epsilon^{2} f \ll 1$, and viscous normal stresses when $\epsilon^{2} \sin ^{2} \alpha \ll 1$. Thus, $\theta \approx \theta_{\text {stat }}$ in $x-y$-planes.

(b) Gravity effects in all directions are neglected, $B o_{z} \equiv f \rho g_{z} H L / \sigma \ll 1$ and $B o_{x y} \equiv$ $f \rho g_{x y} H^{2} / \sigma \ll 1$.

(c) In the vicinity of the bulk meniscus the proportion of the total cross-sectional area occupied by the fluid in the corner is small. This is the low saturation limit $\beta /(1-\beta) \ll 1$, where $\beta \equiv F_{A} H^{2} / A_{s} \ll 1$.

\subsubsection{Bulk meniscus assumptions}

(a) The volume of gas $V_{b}$ in the bulk meniscus curvature region is small, $V_{b} / L A_{s} \ll 1$. Bulk menisci are modelled as flat, $z_{c l}=z_{b}$, figure 5 .

(b) The method of de Lazzer et al. is sufficient to determine the radius of curvature $R$ from which $H=R / f$ in the cross-flow plane at the bulk meniscus location, (3.1). In other words, $z_{2}=z_{b}=z_{c l}$ and $h\left(z_{2}(t)\right)=H$. It is further argued herein with the support of experiments that weakly $z$-dependent geometries may also be modelled using this method, where $h\left(z_{2}(t)\right)=H(z)$ provided the characteristic container edge taper angle $\psi$, to be defined shortly, is small; namely, $\psi^{2} \ll 1$.

(c) Viscous normal stresses are low at the bulk meniscus such that dynamic contact angle effects may be neglected there as well, $C a_{b} \equiv \mu W_{b} / \sigma \sim \epsilon \beta \sin ^{2} \alpha / f \ll 1$, where $W_{b}$ is the characteristic velocity of the bulk meniscus. Thus, $\theta \approx \theta_{\text {stat }}$ everywhere. This criterion must remain satisfied for imposed flows at bulk menisci as well and may be readily assessed $a$ posteriori.

\subsection{Edge flow review}

As a result of these assumptions, for $\epsilon^{2} \ll 1$ the zeroth-order Navier-Stokes equations reduce to the $z$-component equation only

$$
\frac{1}{\mu} \frac{\partial P}{\partial z}=\frac{\partial^{2} w}{\partial x^{2}}+\frac{\partial^{2} w}{\partial y^{2}},
$$

subject to no-slip along the walls and no shear stress on the free surface. Pressure and surface curvature are determined from the normal stress boundary condition and contact angle condition such that

$$
P=-\frac{\sigma}{r}=-\frac{\sigma}{f h},
$$

where $r=r(z, t)$ is the local radius of interface curvature, $h=h(z, t)$, and the entire free surface $S(y, z, t)$, identified in the sectional view of figure $5(b)$, may be expressed as a collection of circular arcs given by

$$
S=h(1+f)-\left((f h)^{2}-y^{2}\right)^{1 / 2}
$$

for $0<y \leqslant f h \sin \delta \equiv y_{m}$ (refer to sections in figures $3 b$ and $5 b$ for notation). 
Following the Laplacian scaling method detailed by Weislogel, Chen \& Bolledulla (2008), (3.3) is non-dimesionalized using the $z$ - and $t$-dependent scales

$$
\begin{aligned}
x, S \sim h & \equiv \bar{x}_{s}, \\
y \sim h \tan \alpha & \equiv \bar{y}_{s},
\end{aligned}
$$

where an overbar is used to distinguish these scales and the quantities that are nondimensionalized by them. From (3.3), a $z$ - and $t$-dependent average velocity scale is determined to be

$$
w \sim \bar{w}_{s} \equiv-\frac{\bar{x}_{s}^{2}}{\mu} \frac{\partial P}{\partial z}\left(\frac{\bar{T}_{c}^{2}}{1+\bar{T}_{c}^{2}}\right),
$$

where $\bar{T}_{c}=\bar{y}_{s} / \bar{x}_{s}(=\tan \alpha$ in this case). Thus, the relevant dimensionless dependent and independent variables for the cross-flow velocity profile are

$$
\bar{x}^{*}=x / \bar{x}_{s}, \quad \bar{y}^{*}=y / \bar{y}_{s}, \quad \bar{w}^{*}=w / \bar{w}_{s} .
$$

Substitution of these variables into (3.3) yields a modified Poisson's equation for the dimensionless $z$-momentum balance

$$
1=\left(\frac{\bar{T}_{c}^{2}}{1+\bar{T}_{c}^{2}}\right) \frac{\partial^{2} \bar{w}^{*}}{\partial \bar{x}^{* 2}}+\left(\frac{1}{1+\bar{T}_{c}^{2}}\right) \frac{\partial^{2} \bar{w}^{*}}{\partial \bar{y}^{* 2}}=\sin ^{2} \alpha \frac{\partial^{2} \bar{w}^{*}}{\partial \bar{x}^{* 2}}+\cos ^{2} \alpha \frac{\partial^{2} \bar{w}^{*}}{\partial \bar{y}^{* 2}} .
$$

Equation (3.10) can be solved and integrated to find the average velocity

$$
\left\langle\bar{w}^{*}\right\rangle=\frac{\bar{x}_{s} \bar{y}_{s}}{A} \int_{0}^{\bar{y}_{m}^{*}} \int_{\bar{y}^{*}}^{\bar{s}^{*}} \bar{w}^{*} \mathrm{~d} \bar{x}^{*} \mathrm{~d} \bar{y}^{*}
$$

where $A$ is the dimensional flow area given by

$$
A=F_{A} h^{2},
$$

with $F_{A}$ defined in (3.2) for a sharp edge. For sharp edges the dimensionless average velocity coefficient is bounded such that $1 / 8 \lesssim\left\langle\bar{w}^{*}\right\rangle \leqslant 1 / 6$ for all values of $\alpha$ and $\theta$. For analytical purposes it is often treated as an $O(1)$ constant. The exact value can be obtained from the numerical results of Bolleddula (2007) where $\left\langle\bar{w}^{*}\right\rangle$ is equivalent to $F_{i}$.

The local average velocity may in turn be used to determine the flow along the edges. Introducing the edge flow capillary velocity coefficient $\mathscr{W} \equiv \sigma\left\langle\bar{w}^{*}\right\rangle \sin ^{2} \alpha / \mu f$, converting back to the dimensional form of (3.11) one finds

$$
\langle w\rangle=\left\langle\bar{w}^{*}\right\rangle \bar{w}_{s}=-\mathscr{W} \frac{\partial h}{\partial z} .
$$

This form may be employed in the dimensional mass-balance equation

$$
\frac{\partial A}{\partial t}=-\frac{\partial(A\langle w\rangle)}{\partial z} .
$$

Finally, substitution of (3.12) and (3.13) into (3.14) yields the local governing dimensional edge flow evolution equation

$$
\frac{\partial h^{2}}{\partial t}=\mathscr{W} \frac{\partial}{\partial z}\left(h^{2} \frac{\partial h}{\partial z}\right)
$$


Dynamical Dirichlet boundary conditions are applied where in general $h\left(z_{1}(t)\right)=H_{1}$ and $h\left(z_{2}(t)\right)=H_{2}$, where $H_{i}$ is either specified or computed via (3.1) if at a bulk meniscus.

\section{Draining flows}

Because draining of the edge wetting fluid means the filling of the non-wetting fluid, such flows might also be termed 'filling' flows depending on the fluid of interest.

\subsection{Formulation}

Beginning with the fixed location draining flow problem of figure $5(b)$ for a uniformsection container with $m=1$ wetted edge, subscripts 1 and 2 refer to quantities evaluated at the origin and bulk meniscus, respectively. The boundary conditions for the edge flow equation (3.15) are

$$
h(0, t)=0 \quad \text { and } \quad h\left(z_{2}(t)\right)=H_{2},
$$

where $\theta<\pi / 2-\alpha$. For this flow type, $z_{1}(t)=0$, where the drain or sink location condition $h\left(z_{1}\right)=H_{1}=0$ is assumed for simplicity.

Employing (3.12) and (3.13),

$$
A\langle w\rangle=-\mathscr{W} F_{A} h^{2} \frac{\partial h}{\partial z} .
$$

A volumetric flow rate balance may be written at the receding bulk meniscus $z_{2}(t)$, where

$$
\left.\left(\mathscr{W} F_{A} h^{2} \frac{\partial h}{\partial z}\right)\right|_{z_{2}}=\left.\frac{\mathrm{d} z_{2}}{\mathrm{~d} t}\left(A_{s}-F_{A} h^{2}\right)\right|_{z_{2}},
$$

subject to $z_{2}(0)=0$. A known initial bubble length or volume could alternatively have been specified.

The two independent variables and one dependent variable of the problem are normalized by the following scales:

$$
z \sim L, \quad h \sim H_{L}, \quad t \sim \frac{A_{s} L}{Q_{s}}, \quad Q_{s}=\frac{F_{A} \mathscr{W} H_{L}^{3}}{L},
$$

where $L$ is either an arbitrary or selectable geometry-dependent length scale. $Q_{s}$ is a total flow rate scale and $W=\mathscr{W} H_{L} / L$ is the average edge flow velocity scale. Normalizing the governing edge flow equation (3.15) by these scales such that

$$
z^{*}=z / L, \quad h^{*}=h / H_{L}, \quad t^{*}=Q_{s} t / A_{s} L, \quad Q^{*}=Q / Q_{s},
$$

gives

$$
\beta \frac{\partial h^{* 2}}{\partial t^{*}}=\frac{\partial}{\partial z^{*}}\left(h^{* 2} \frac{\partial h^{*}}{\partial z^{*}}\right),
$$

subject to boundary conditions $h^{*}\left(z_{1}^{*}=0\right)=H_{1}^{*} \equiv 0$ and $h^{*}\left(z_{2}^{*}\right)=H_{2}^{*} \equiv 1$. The parameter $\beta=F_{A} H_{2}^{2} / A_{s}$ is the ratio of edge flow to bulk meniscus flow time scales, which is equivalent to the local or sectional saturation of the container. The condition $\beta \ll 1$ signals the quasi-steady flow scenario of fast edge flows subject to boundary conditions from slowly developing bulk meniscus flows. 
Using the scales of (4.4) the dimensionless volume balance at the bulk meniscus (4.3) becomes

$$
\left.\frac{\partial h^{*}}{\partial z^{*}}\right|_{z_{2}^{*}}=(1-\beta) \frac{\mathrm{d} z_{2}^{*}}{\mathrm{~d} t^{*}},
$$

subject to $z_{2}^{*}(0)=0$, and where $h^{*}\left(z_{2}^{*}\right)=1$ has been applied.

\subsection{Constant-area draining similarity solution}

Equations (4.6) and (4.7) yield a similarity solution under the diffusive power law transformation

$$
h^{*}=F(\eta), \quad \eta=z^{*} \sqrt{3 / 2 t^{*}}, \quad \eta_{2} \equiv z_{2}^{*} \sqrt{3 / 2 t^{*}},
$$

where $F$ and $\eta$ are dimensionless similarity variables. Upon back-substitution, the local edge flow equation (4.6) becomes

$$
\left(F^{2} F^{\prime}\right)^{\prime}+\frac{2 \beta}{3} \eta F F^{\prime}=0,
$$

subject to $F(0)=0$ and $F\left(\eta_{2}\right)=1$, and the volumetric flow rate balance at the bulk meniscus (4.7) reduces to a slope condition $F^{\prime}\left(\eta_{2}\right)=\eta_{2}(1-\beta) / 3$. Analytical and numerical solutions to (4.9) are aided by noting that the equation is invariant under the transformation

$$
F=\lambda^{2} F^{+}, \quad \eta=\lambda \eta^{+}, \quad \eta_{2}=\lambda \eta_{2}^{+},
$$

which provides the flexibility of specifying $\eta_{2}^{+}$at will. Choosing $\eta_{2}^{+}=1, \lambda=\eta_{2}$, the invariant system becomes

$$
\left(F^{+2}{F^{+\prime}}^{\prime}\right)^{+}+\frac{2 \beta}{3} \eta^{+} F^{+} F^{+^{\prime}}=0,
$$

subject to $F^{+}(0)=0, F^{+}(1)=1 / \lambda^{2}$, and $F^{+^{\prime}}(1)=(1-\beta) / 3$. Numerical solutions for both $F^{+}\left(\eta^{+}\right)$and $F(\eta)$ are presented in figure $6(a, b)$ for various values of $\beta$. Numerical values for the dimensionless bulk meniscus location $\eta_{2}$ and removed flow rate $\left.\left(F^{2} F^{\prime}\right)\right|_{0}$ are presented as functions of $\beta$ in figure $6(c, d)$.

An asymptotic analytic solution to (4.11) is possible by applying the quasi-steady assumption $\beta \ll 1$. Introducing the naive expansions

$$
\begin{aligned}
& F^{+}=F_{0}^{+}+\beta F_{1}^{+}+\beta^{2} F_{2}^{+}+\beta^{3} F_{3}^{+}+\cdots, \\
& \eta_{2}^{+}=\eta_{20}^{+}+\beta \eta_{21}^{+}+\beta^{2} \eta_{22}^{+}+\beta^{3} \eta_{23}^{+}+\cdots,
\end{aligned}
$$

and substituting into (4.11), the $O(1)$ solution is quickly found:

$$
F_{0}^{+}=\eta^{+1 / 3} \text {. }
$$

The coefficient $(3 / 2)^{1 / 2}$ defining $\eta$ in (4.8) is conveniently chosen such that

$$
\eta_{20}=\eta_{20}^{+}=1
$$

After substitution of $F_{0}^{+}$and $\eta_{20}^{+}$into the expanded (4.11), it is observed that each of the higher-order equations are inhomogeneous Euler-Cauchy equations of the form

$$
9 \eta^{+2}{F_{i}^{+\prime}}^{\prime \prime} 12 \eta^{+}{F_{i}^{+}}^{\prime}-2 F_{i}^{+}=f c n\left(\eta^{+}\right), \quad i=1,2, \ldots,
$$



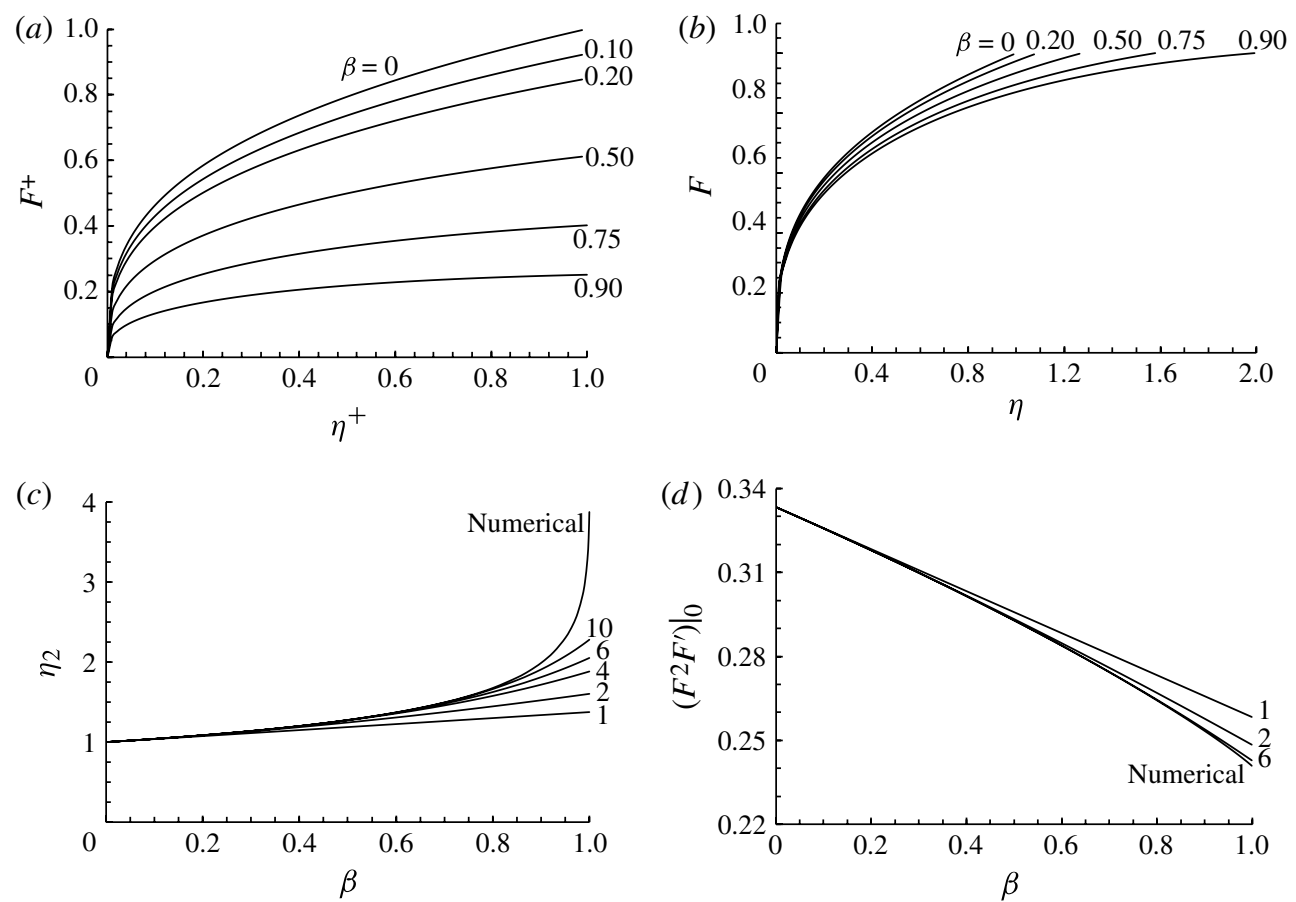

FIgURE 6. (a) Exact numerical solutions to (4.11) for $F^{+}\left(\eta^{+}\right)$and $(b)$ back-transformed function $F(\eta)$ for select values of $\beta$. (c) Numerical solutions for $\eta_{2}(\beta)$ (三 $\lambda$ ) from (4.11) and $(d)$ back-transformed numerical solution for flow rate function $\left.\left(F^{2} F^{\prime}\right)\right|_{0}$. Asymptotic solutions to indicated order of $\beta$ are also provided for comparisons in $(c)$ and $(d)$ using (4.18) and (4.19), respectively: e.g. '2' implies a solution accurate to $O\left(\beta^{2}\right)$.

with homogeneous slope conditions $F_{i}^{+^{\prime}}(1)=0$ for $i=2,3, \ldots$ These linear equations are readily integrated to any order, which suggests that an exact infinite series solution for all $\beta$ of the form $F^{+}=\sum_{i=0}^{\infty} C_{i} \eta^{+(1+5 i) / 3}$ is possible, where $C_{i}=C_{i}(\beta)$. Such a solution was found, but will be reported separately. Sticking with the equivalent asymptotic method, successive solutions to $O\left(\beta^{4}\right)$ in terms of $F(\eta)$ yield

$$
\begin{aligned}
h^{*}= & F=\eta^{1 / 3}-\beta\left(\frac{3 \eta^{1 / 3}}{40}+\frac{\eta^{2}}{20}\right)-\beta^{2}\left(\frac{129 \eta^{1 / 3}}{8320}-\frac{3 \eta^{11 / 3}}{1040}\right) \\
& -\beta^{3}\left(\frac{15811 \eta^{1 / 3}}{299520}-\frac{9 \eta^{11 / 3}}{41600}+\frac{7 \eta^{16 / 3}}{187200}\right)-\beta^{4}\left(\frac{111926173 \eta^{1 / 3}}{477634560000}\right. \\
& \left.-\frac{2637 \eta^{11 / 3}}{43264000}+\frac{7 \eta^{16 / 3}}{1248000}+\frac{1091 \eta^{7}}{186576000}\right)+O\left(\beta^{5}\right)
\end{aligned}
$$

and

$$
\eta_{2}=1+\frac{3 \beta}{8}+\frac{375 \beta^{2}}{1664}+\frac{485 \beta^{3}}{3072}+\frac{15304397 \beta^{4}}{127369216}+O\left(\beta^{5}\right),
$$

from which the flow rate removed from the conduit may be computed by

$$
\left.\left(F^{2} F^{\prime}\right)\right|_{0}=\frac{1}{3}-\frac{3 \beta}{40}-\frac{411 \beta^{2}}{41600}-\frac{46331 \beta^{3}}{14976000}-\frac{22263213 \beta^{4}}{15921152000}+O\left(\beta^{5}\right) .
$$


Higher-order expansions are readily obtained and solutions for $\eta_{2}$ and $\left.F^{2} F^{\prime}\right|_{0}$ are listed in the Appendix to $O\left(\beta^{10}\right)$. The asymptotic solutions (4.17)-(4.19) are extended to $O\left(\beta^{10}\right)$ and compared to the numerical solutions in figure $6(c, d)$. It is clear from the signs of the terms in (4.17)-(4.19) that the higher orders account for the liquid volume along the edge by reducing $h^{*}=F(\eta)$ and increasing $\eta_{2}$. The net effect is one of reducing the fluid withdrawal rate $\left(\left.F^{2} F^{\prime}\right|_{0}\right)$ along the edges by decreasing the capillary driving force (slope) and increasing the viscous resistance (length). Inspection of figure 6(c) reveals excellent agreement of numerical and asymptotic values of $\eta_{2}$ for $\beta \lesssim 0.5$, but that as $\beta \rightarrow 1$ errors increase to $>40 \%$ despite a 10th-order-accurate approximation. It is also valuable to note from (4.19) and figure $6(d)$ that flow rates are much less dependent on $\beta$, even when $\beta \sim 1$. In fact, in the unphysical situation $\beta=1$, the second-order correction is $<3 \%$ of the $O(1)$ solution. Further, it can be shown that the first-order solution of (4.19) for $\beta=1$ differs by only $\approx 6 \%$ of a 6th-order solution, the latter being nearly coincident with the exact numerical solution presented in figure $6(d)$. Such observations will be invoked later to explain how even the $O$ (1) solutions are capable of identifying optimal flow rate geometries when $\beta \ll 1$ is not necessarily true.

In terms of $z^{*}$ and $t^{*}$

$$
z_{2}^{*}=\eta_{2}\left(2 t^{*} / 3\right)^{1 / 2}
$$

and at $z=\eta=0$

$$
Q^{*}(0)=\left.\left(3 / 2 t^{*}\right)^{1 / 2}\left(F^{2} F^{\prime}\right)\right|_{0} .
$$

\subsection{Generalization}

The above analysis applies well to the single edge flow scenario of figures 1 and 5, but containers with arbitrary- $m$ wetted edges require additional bookkeeping. For example, the constant-area draining flow governing system requires an altered scaling for each $j$ th edge flow. The local interface height, bulk meniscus time, and total flow rate scales are expressed as

$$
h_{j} \sim H_{L j}, \quad t \sim \frac{A_{s L} L}{Q_{s}}, \quad Q_{s}=\sum_{j=1}^{m} F_{A j} \mathscr{W}_{j} H_{L j}^{3} / L,
$$

respectively. Subscripts $L$ on $H_{L j}, P_{s L}$, and $A_{s L}$ denote $H_{j}, P_{s}$, and $A_{s}$ scales evaluated at length scale $z \sim z_{2} \sim L$. The normalized flow area function $\Sigma$, edge flow area function $F_{A}$, and curvature function $f$ also depend on the $j$ th corner characteristics and (3.2) becomes

$$
\Sigma=\sum_{j=1}^{m} \frac{F_{A j}}{f_{j}^{2}}, \quad F_{A j}=f_{j}^{2}\left(\frac{\cos \theta \sin \delta_{j}}{\sin \alpha_{j}}-\delta_{j}\right), \quad f_{j}=\frac{\sin \alpha_{j}}{\cos \theta-\sin \alpha_{j}},
$$

where $\delta_{j} \equiv \pi / 2-\alpha_{j}-\theta$. Normalizing (3.15) by these scales the $j$ th edge flow equation becomes

$$
\left(\sum_{j=1}^{m} \beta_{j} \omega_{j}\right) \frac{\partial h_{j}^{* 2}}{\partial t^{*}}=\omega_{j} \frac{\partial}{\partial z^{*}}\left(h_{j}^{* 2} \frac{\partial h_{j}^{*}}{\partial z^{*}}\right)
$$

subject to the boundary conditions $h^{*}\left(z_{1}^{*}=0\right)=H_{1}^{*} \equiv 0$ and $h^{*}\left(z_{2}^{*}\right)=H_{2}^{*} \equiv 1$, with two parameters

$$
\beta_{j}=A_{j} / A_{s}, \quad \omega_{j}=\mathscr{W}_{j} / \mathscr{W}_{m},
$$


(a)

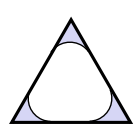

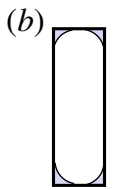

(c)

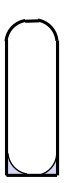

$(d)$

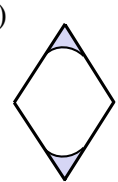

(e)

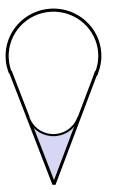

$(f)$

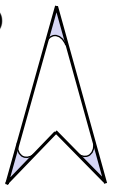

$(g)$

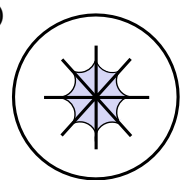

(h)

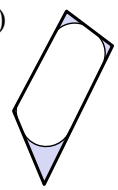

FIGURE 7. (Colour online) Selection of applicable container sections: (a) n-regular polygons, $(b)$ rectangles, $(c)$ combined rounded rectangles, $(d)$ selective but equal edge wetting sections, (e) $m=1$ sections, $(f)$ certain re-entrant sections, $(g)$ vane structures, and $(h)$ irregular polygonal sections. Sections $(a-g)$ depict $m$-regular edge wetting geometries. Proportionally tapered containers of such section types may be analysed by the current methods if the effective taper angle $\psi^{2} \ll 1$.

where $\mathscr{W}_{m}$ is the maximum of the $m$ local average velocity coefficients. The quantity $\sum_{j=1}^{m} \beta_{j} \omega_{j}$ appearing on the left-hand side of (4.24) is again a ratio of total edge flow to bulk meniscus flow time scales. Appearing alone, $\beta_{j}$ is the ratio of the $j$ th edge flow to the bulk meniscus time scale. The condition $\sum_{j=1}^{m} \beta_{j} \omega_{j} \ll 1$ is required to satisfy the quasi-steady flow assumption. The parameter $\omega_{j}$ is a ratio of the $j$ th velocity scale to the maximum velocity scale, thus $0<\omega_{j} \leqslant 1$. When $\omega_{j} \ll 1$ the $j$ th corner flow does not contribute significantly to the overall flow rate and may be ignored at $O(1)$. Using the altered scales of (4.22), the dimensionless volume balance (4.3) at the bulk meniscus $z_{2}^{*}$ becomes

$$
\frac{\left.\sum_{j=1}^{m} \beta_{j} \omega_{j}\left(h_{j}^{* 2} \frac{\partial h_{j}^{*}}{\partial z^{*}}\right)\right|_{z_{2}^{*}}}{\sum_{j=1}^{m} \beta_{j} \omega_{j}}=\left.\frac{\mathrm{d} z_{2}^{*}}{\mathrm{~d} t^{*}}\left(A_{s}^{*}-\sum_{j=1}^{m} \beta_{j} h_{j}^{* 2}\right)\right|_{z_{2}^{*}} .
$$

The limiting case of $\beta_{j} \ll 1$ represents flows of low saturation and the liquid volume occupying the corners may be ignored at $O(1)$. The parameter $\beta_{j} \omega_{j} / \sum_{j=1}^{m} \beta_{j} \omega_{j}$ appears in each of the $m$ terms on the left-hand side of (4.26) and represents the ratio of the $j$ th edge flow rate to total flow rate.

The forms of (4.24)-(4.26) are greatly simplified for the special case of identical corner flow velocity scales. For such containers $\omega_{j}=1$. A further simplification results if the cross-flow area is also identical for each corner. Thus, for uniform contact angle and equal interior edge angles, the edge flow and global volume balance equations reduce to the previous forms, (4.6) and (4.7), only now $\beta=m \beta_{j}$. Such containers are referred to as ' $m$ regular' sections where each wetted interior edge is geometrically identical. Sample sections satisfying this condition are sketched in figure $7(a-g)$, the $m=1$ section being a special case. The $m$-regular simplification is not necessary to yield solutions, as in the case of irregular sections such as figure 7(h), but $m$-regularity is adopted here mostly for clarity and on occasion when certain example problems are readily comparable to practical geometries.

\subsection{Extension to tapered sections}

The above analytic approach may be extended to sections with weak $z$-dependence as sketched in figure 8 for an $m=1$ section. A straight-edge taper where $A_{s} \propto z^{2}$ is shown in figure $8(a, b)$. An observed flow is depicted in figure $8(a)$ with model interface profile sketched in figure $8(b)$. An arbitrary power law taper is sketched in $8(c)$, where $A_{s} \propto z^{2 n}$. In reference to figure $8(a)$, the solutions depend on a weak taper assumption where locally $z=z^{\prime}+O\left(\psi^{2}\right)$, with $\psi$ being a local expanding or 
(a)

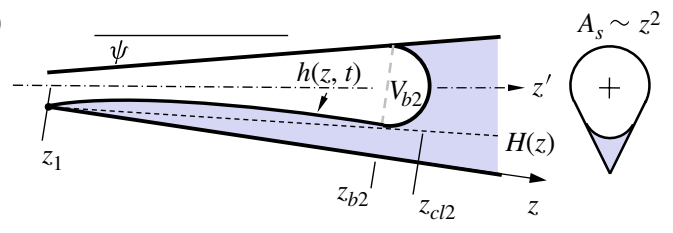

(b)

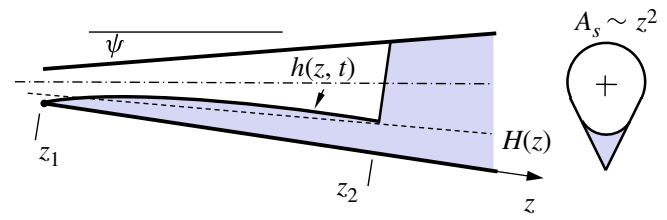

(c)

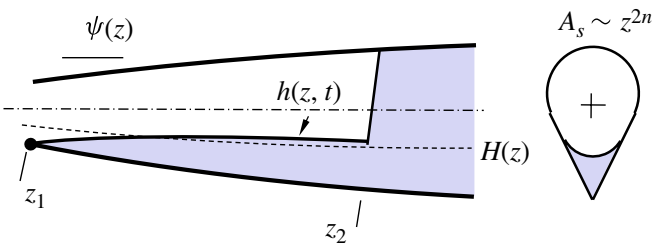

FIgURE 8. (Colour online) Schematics of fixed location draining at $z_{1}$ for an $m=1$ section: (a) parabolic taper of angle $\psi,(b)$ model of flow in $(a)$, and $(c)$ power law taper where $\psi=\psi(z)$. The model employs the dynamical boundary condition $h\left(z_{2}, t\right)=H(z)$ in $(b)$ and $(c)$.

contracting taper angle. When $\psi^{2} \ll 1$, the bulk meniscus curvature condition of (3.1) may be written in the form of $A_{s}(z)$ and $P_{s}(z)$ for what is considered here as a local de Lazzer et al. dynamical boundary condition for $H_{j}(z)$ at bulk menisci,

$$
H_{j}(z)=\frac{R}{f_{j}}=\frac{P_{s} \cos \theta}{2 f_{j} \Sigma}\left(1-\left(1-\frac{4 \Sigma A_{s}}{P_{s}^{2} \cos ^{2} \theta}\right)^{1 / 2}\right),
$$

with $\Sigma, F_{A j}$ and $f_{j}$ given in (4.23). Again, assuming an $m$-regular section, the $j$ edge flow equations are identical,

$$
\beta \frac{\partial h^{* 2}}{\partial t^{*}}=\frac{\partial}{\partial z^{*}}\left(h^{* 2} \frac{\partial h^{*}}{\partial z^{*}}\right)
$$

subject to $h^{*}(0)=0$ and $h^{*}\left(z_{2}^{*}\right)=H^{*}\left(z_{2}^{*}\right)$ and the bulk meniscus flow rate balance at $z_{2}^{*}$,

$$
\left.\left(h^{* 2} \frac{\partial h^{*}}{\partial z^{*}}\right)\right|_{z_{2}^{*}}=\left.\frac{\mathrm{d} z_{2}^{*}}{\mathrm{~d} t^{*}}\left(A_{s}^{*}-\beta H^{* 2}\right)\right|_{z_{2}^{*}}
$$

subject to initial condition $z_{2}^{*}(0)=0$.

Assuming $\beta \ll 1$, the $O(1)$ solution to (4.28) and (4.29) yields

$$
h_{0}^{*}=H^{*}\left(z_{20}^{*}\right)\left(\frac{z^{*}}{z_{20}^{*}}\right)^{1 / 3}
$$

and

$$
t^{*}=3 \int \frac{A_{s}^{*}}{H^{* 3}} z_{20}^{*} \mathrm{~d} z_{20}^{*}
$$

These forms may be solved for arbitrary taper type provided $\psi^{2} \ll 1$ locally. 
In general, solutions for $z_{20}^{*}$ are implicit in $t^{*}$. To proceed with the analysis from here one must restrict the discussion to a specific container geometry which then specifies $A_{s}(z), P_{s}(z)$, and $H_{j}(z)$. By inspection of (4.27), at least two unique simplifying situations occur. First, when $A_{s} / P_{s}^{2}=$ constant $\equiv k^{2}, H \propto P_{s}\left(\right.$ or $\left.H \propto A_{s}^{1 / 2}\right)$. Second, when the fractional argument in the radical is small it can be shown that to zeroth order $H \propto A_{s} / P_{s}$. We will focus on the former where (4.27) is re-written

$$
H_{j}(z)=\frac{P_{s} \cos \theta}{2 f_{j} \Sigma}\left(1-\left(1-\frac{4 \Sigma k^{2}}{\cos ^{2} \theta}\right)^{1 / 2}\right) \equiv \frac{F_{H P} P_{s}}{f_{j}}=\frac{F_{H P} A_{s}^{1 / 2}}{f_{j} k},
$$

where $F_{H P}$ collects many of the geometric quantities of $H_{j}(z)$ into a single term. Many common geometries such as squares, rectangles, rhombi and others can possess this ' $k{ }^{2}$ property' provided they taper proportionally (see figure 7). In the case $A_{s} / P_{s}^{2}=k^{2}$, for $m$-regular containers, after non-dimensionalizing $H$ by $H_{L}=F_{H P} A_{L}^{1 / 2} / f_{j} k$, substitution of (4.32) into (4.31) yields

$$
t^{*}=3 \int \frac{z_{20}^{*}}{A_{s}^{* 1 / 2}} \mathrm{~d} z_{20}^{*},
$$

which can be solved for a wide variety of container taper types. Here we consider a power law taper of the form $A_{s}=A_{L} z^{* 2 n}$ sketched in figure 8(c), which produces

$$
H^{*}=z^{* n}, \quad A_{s}^{*}=z^{* 2 n} .
$$

Thus, (4.33) may be solved to find that

$$
z_{20}^{*}=\left(\frac{(2-n) t^{*}}{3}\right)^{1 /(2-n)}, \quad Q^{*}=\frac{1}{3}\left(\frac{(2-n) t^{*}}{3}\right)^{(3 n-1) /(2-n)}, \quad n \neq 2,
$$

and

$$
z_{20}^{*}=\exp \left[\frac{t^{*}}{3}\right] \quad Q^{*}=\frac{1}{3} \exp \left[\frac{5 t^{*}}{3}\right], \quad n=2 .
$$

For $n \geqslant 2, z_{20}^{*}$ is singular as $\tau^{*} \rightarrow 0$. It is easy to determine the forms for various $n$, and expressions for constant, 2/3-power, linear, and parabolic area conduits are

$$
\begin{gathered}
n=0, \quad A_{s}^{*}=1, \quad z_{20}^{*}=\left(\frac{2 t^{*}}{3}\right)^{1 / 2}, \quad Q^{*}=\frac{1}{3}\left(\frac{2 t^{*}}{3}\right)^{-1 / 2}, \\
n=\frac{1}{3}, \quad A_{s}^{*}=z^{* 2 / 3}, \quad z_{20}^{*}=\left(\frac{5 t^{*}}{9}\right)^{3 / 5}, \quad Q^{*}=\frac{1}{3}, \\
n=\frac{1}{2}, \quad A_{s}^{*}=z^{*}, \quad z_{20}^{*}=\left(\frac{t^{*}}{2}\right)^{2 / 3}, \quad Q^{*}=\frac{1}{3}\left(\frac{t^{*}}{2}\right)^{1 / 3}, \\
n=1, \quad A_{s}^{*}=z^{* 2}, \quad z_{20}^{*}=\frac{t^{*}}{3}, \quad Q^{*}=\frac{t^{* 2}}{9} .
\end{gathered}
$$

The case $n=1$ is the straight edge taper sketched in figure $8(a, b)$ and the case $n=0$ recovers the constant-area draining solutions of $\S 4.2$. Contracting and expanding tapers may be modelled by forms such as $A_{s}^{*}\left(z_{2}^{*}\right) \sim 1 \pm c z_{20}^{* 2 n}$, and so on. Many other functional forms may be suggested or dictated by applications. 
(a)

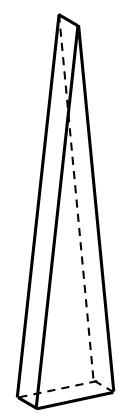

(b)

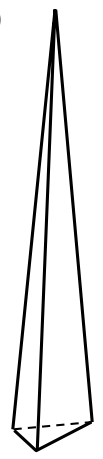

(c)

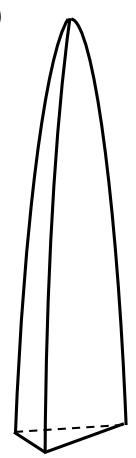

(d)

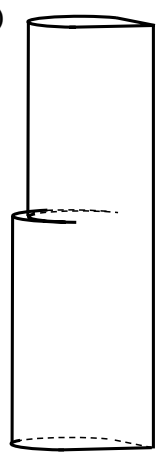

(e)

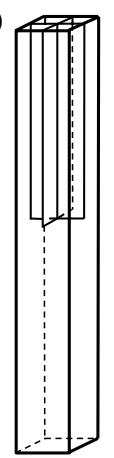

$(f)$

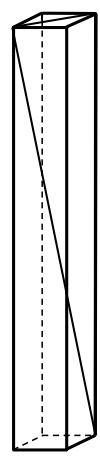

$(g)$

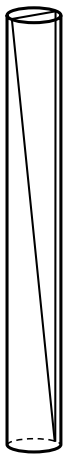

FIGURE 9. Depiction of several container taper types for bubble displacement flows: $(a)$ linear, $(b)$ parabolic, $(c)$ power law, $(d)$ stepped $m=1$ container, $(e)$ stepped square, and $(f)$ square and $(g)$ circular conduits with tapered vane structures.

\subsection{Comparison with experiments}

Combining results from $\S \S 4.2$ and 4.3 , (4.20) with $m=4, \beta=4 \beta_{j}$, and $\mathscr{W}=4 \mathscr{W}_{j}$ is readily compared with the $m$-regular experimental constant area square tube draining results introduced in figures 3 and 4. From (4.20) (see also (4.37)), the dimensional sharp corner solution for the receding bulk meniscus is

$$
z_{2}=\left(\frac{2 m F_{A} \mathscr{W} H^{3}}{3 A_{s}} t\right)^{1 / 2}\left(1+\frac{3 \beta}{8}+\frac{375 \beta^{2}}{1664}+\cdots\right)
$$

Accounting for the presence of rounded interior edges (see Chen et al. 2006, where for this nominally $1 \mathrm{~mm}$ thin-walled rounded square tube and fluid: inner diameter $=1.02 \mathrm{~mm}, R_{c} \approx 0.12 \mathrm{~mm}, A_{s}=0.975 \mathrm{~mm}^{2}, P_{s}=3.77 \mathrm{~mm}, \lambda=0.456,\left\langle\bar{w}^{*}\right\rangle=0.1729$, and $\beta=0.067)$ the asymptotic prediction is well within the scatter of the repeat experiments shown in figure $3(c)$. Note that in these comparisons $z_{2}=z_{c l}$ is assumed and that the higher-order terms contribute negligibly to this solution. Such favourable preliminary agreement encourages extension of the method to problems of increased geometric complexity.

\section{Bubble displacement}

\subsection{Formulation}

A variety of solutions may be similarly developed for slender tapered geometries with finite length or volume scales that serve as models for bubble motion in weakly three-dimensional containers due to edge flows. A selection of applicable container families is sketched in figure 9. The example weakly expanding containers depicted in figure $9(b-d)$ are modelled in figure 10 for the $m=1$ section with taper angle $\psi$ which is exaggerated for clarity. The taper yields a passive capillary displacement of the bubble away from the narrow region of the section as liquid is pumped along the edges by a pressure gradient due to differences in bulk meniscus curvature - high bulk meniscus curvature at $z_{1}^{*}$ and low bulk meniscus curvature at $z_{2}^{*}$. Figure $10(b, c)$ provide sketches of the model problems. The stepped taper geometry idealized in figure $10(d)$ will be addressed in $\S 6.3$. Equation (4.28) remains applicable but is now subject to $H_{1}^{*}\left(z_{1}^{*}\right)$ and $H_{2}^{*}\left(z_{2}^{*}\right)$. Equation (4.29) applies at $z_{2}^{*}$, but a volume balance must also apply 
(a)

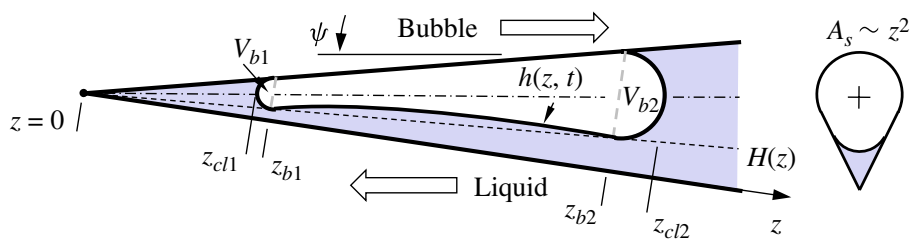

(b)

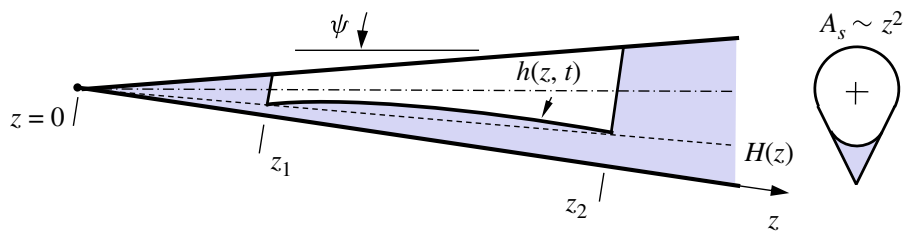

(c)

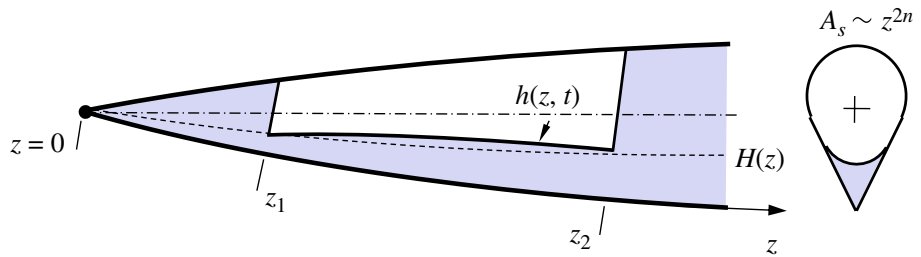

(d)

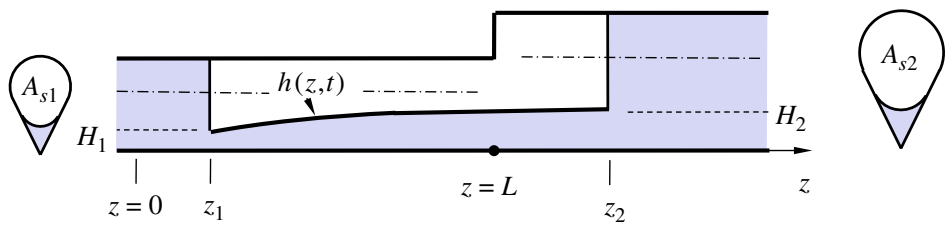

FIGURE 10. (Colour online) Schematic of bubble displacement flows for $A_{s} / P_{s}^{2}=k^{2}=$ constant tapers: $(a)$ sketch of actual flow for $A_{s} \sim z^{2}$ taper with edge flow and bubble motion identified with arrows, $(b)$ model flow for taper in $(a),(c)$ power law taper where $A_{s} \sim z^{2 n}$, and (d) stepped taper.

at $z_{1}^{*}$; namely,

$$
\left.\left(h^{* 2} \frac{\partial h^{*}}{\partial z^{*}}\right)\right|_{z_{1}^{*}}=\left.\frac{\mathrm{d} z_{1}^{*}}{\mathrm{~d} t^{*}}\left(A_{s}^{*}-\beta H^{* 2}\right)\right|_{z_{1}^{*}}
$$

To conserve the gas volume, a bubble volume constraint is also employed

$$
\frac{V_{u}}{A_{s L} L}=\int_{z_{1}^{*}}^{z_{2}^{*}}\left(A_{s}^{*}-\beta h^{* 2}\right) \mathrm{d} z^{*},
$$

where $V_{u}$ represents the fixed bubble volume. The system of equations (4.28), (4.29), (5.1), and (5.2) may be solved to determine key characteristics of the flow.

\subsection{Power law tapers, $A_{s} \sim z^{2 n}$ : exact solution}

For power law tapers of the form of (4.34), for $\beta \ll 1$, the $O$ (1) bubble displacement solution to the dimensionless edge flow (4.28) is

$$
h^{*}=\left[z_{2}^{* 3 n}+\left(\frac{z_{2}^{* 3 n}-z_{1}^{* 3 n}}{z_{2}^{*}-z_{1}^{*}}\right)\left(z^{*}-z_{2}^{*}\right)\right]^{1 / 3} .
$$


The case $n=0$ is the constant-area situation where $A_{s}^{*}=1$, and produces the exact 'no flow' condition where $h^{*}=1$. A second exact solution is observed when $n=1 / 3$, where $A_{s}^{*}=z^{* 2 / 3}$. This unique taper produces edge flow interfaces where

$$
h^{*}=z^{* 1 / 3},
$$

which exactly satifies (4.28). By choosing $L=5 V_{u} / 3 A_{s L}(1-\beta)$, the bubble volume constraint equation (5.2) becomes

$$
z_{2}^{* 5 / 3}=1+z_{1}^{* 5 / 3}
$$

which is a convenient form for initial conditions $z_{1}^{*}(0)=0$ and $z_{2}^{*}(0)=1$, and gives practical values for $H(L)=H_{L}, A_{s}(L)=A_{s L}, R(L)=R_{L}$, etc. Combining (5.1) and (5.5), the bulk menisci locations are determined by

$$
z_{1}^{*}=\left(\frac{5 t^{*}}{9(1-\beta)}\right)^{3 / 5}, \quad z_{2}^{*}=\left(1+\frac{5 t^{*}}{9(1-\beta)}\right)^{3 / 5},
$$

where en route it is learned that the dimensionless flow rate across the bubble is

$$
Q^{*}=h^{* 2} \frac{\partial h^{*}}{\partial z^{*}}=\frac{1}{3} \text {. }
$$

The constant flow rate arises because the interface $h^{*}=z^{* 1 / 3}$ coincides with the local constant height location $H^{*}=z^{* 1 / 3}$. Neither quantities are time-dependent and the edge flow interface profile appears stationary as the bulk menisci advance or recede over it. Mechanistically, the driving force for the flow changes with $H^{*}$ at exactly the same rate the viscous resistance changes with length $z_{2}^{*}-z_{1}^{*}$. The solution is accurate for all orders of $\beta$ because the left-hand side of (4.28) is identically zero and because $A_{s}^{*}=h^{* 2}=H^{* 2}=z^{* 2 / 3}$.

\subsection{Power law tapers, $A_{s} \sim z^{2 n}: O(1)$-accurate solutions}

For $\beta \ll 1$, substitution of (5.3) into (5.1) yields an $O(1)$-accurate flow rate balance at $z_{1}^{*}$

$$
\frac{1}{3}\left(\frac{z_{2}^{* 3 n}-z_{1}^{* 3 n}}{z_{2}^{*}-z_{1}^{*}}\right)=z_{1}^{* 2 n} \frac{\mathrm{d} z_{1}^{*}}{\mathrm{~d} t^{*}} .
$$

The flow rate balance equation (4.29) at $z_{2}^{*}$ is redundant at $O(1)$, but after choosing $L=V_{u}(2 n+1) / A_{s L}$ the bubble volume constraint from (5.2) can be used to write $z_{2}^{*}$ in terms of $z_{1}^{*}$,

$$
z_{2}^{* 2 n+1}=1+z_{1}^{* 2 n+1}
$$

which when substituted into (5.8) provides the implicit integral equation

$$
t^{*}=3 \int \frac{\left(1+z_{1}^{* 2 n+1}\right)^{1 /(2 n+1)}-z_{1}^{*}}{\left(1+z_{1}^{* 2 n+1}\right)^{3 n /(2 n+1)}-z_{1}^{* 3 n}} z_{1}^{* 2 n} \mathrm{~d} z_{1}^{*} .
$$

For a linearly increasing area $n=1 / 2, A_{s}^{*}=z^{*}$, and (5.10) yields the un-integrable equation

$$
t=3 \int \frac{\left(1+z_{1}^{* 2}\right)^{1 / 2}-z_{1}^{*}}{\left(1+z_{1}^{* 2}\right)^{3 / 4}-z_{1}^{* 3 / 2}} z_{1}^{* 2} \mathrm{~d} z_{1}^{*}
$$


(a)

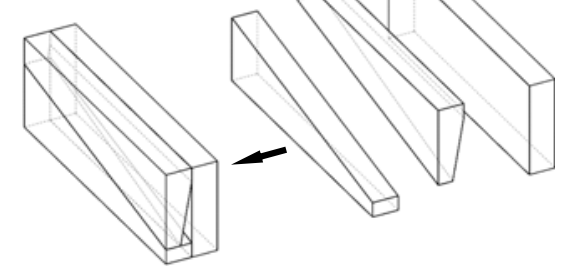

(b)

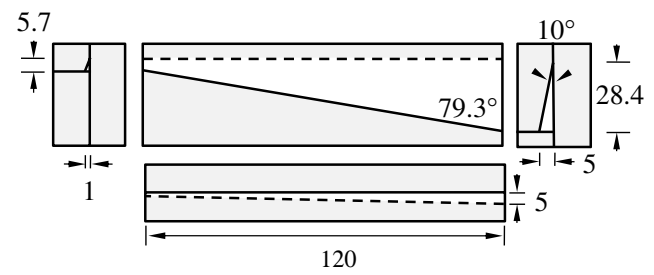

FIGURE 11. Tapered right triangle employed in bubble displacement drop tower test: $(a)$ enlarged view of prismatic elements and $(b)$ dimensions in $\mathrm{mm}$. The test cell is sealed with cellophane tape.

The situation is improved when $n=1$ and $A_{s}^{*}=z^{* 2}$, figure $10(b)$, where (5.10) and (5.9) yield the implicit solution

$$
t^{*}=\frac{1}{4}\left(\left(1+z_{1}^{* 3}\right)^{4 / 3}-z_{1}^{* 4}-1\right), \quad z_{2}^{*}=\left(1+z_{1}^{* 3}\right)^{1 / 3}
$$

from which the flow rate may be determined from the left-hand side of (5.1):

$$
Q^{*}=h^{* 2} \frac{\partial h^{*}}{\partial z^{*}}=\frac{1}{3\left(z_{2}^{*}-z_{1}^{*}\right)} .
$$

For small times, explicit zeroth-order forms are

$$
\begin{gathered}
z_{10}^{*}=t^{* 1 / 3}+\frac{1}{4} t^{* 2 / 3}+\frac{3}{16} t^{*}+O\left(t^{* 4 / 3}\right), \\
z_{20}^{*}=1+\frac{1}{3} t^{*}+O\left(t^{* 4 / 3}\right), \\
Q_{0}^{*}=1+\frac{1}{3} t^{*}+\frac{5}{12} t^{* 2 / 3}+\frac{65}{144} t^{*}+O\left(t^{* 4 / 3}\right) .
\end{gathered}
$$

This solution was first reported by Weislogel et al. (2007).

\subsection{Comparison with experiments}

The solutions of (5.12) for bubble displacements in tapered conduits where $A_{s} / P_{s}^{2}=k^{2}$ and $A_{s} \sim z^{2}$ are qualitatively compared here to drop tower experiments performed at NASA's Glenn Research Center $2.2 \mathrm{~s}$ drop tower, Lekan et al. (1996). In contrast to similar microfluidic experiments $\lesssim O(1) \mathrm{mm}$, the brief near-weightless environment of a drop tower permits the use of significantly larger three-dimensional tapered geometries that are easier to construct with ideal sharp edges and smooth surfaces. The larger size and sudden reduction in body force for the drop tests provide a measure of control of the initial conditions not readily achieved in microfluidic tests. The larger containers also produce significantly faster capillary flows. Fluid properties may be selected to adequately exploit the brief experiment time afforded by the drop.

A weakly tapered triangular section is shown in figure 11 with assembly method and dimensions noted. The acrylic prismatic components are bonded using methylene chloride. Seepage into the interior edges of the vessel is minimal. The container is a skewed 10-80-90 pyramidal frustum and satisfies the $A_{s}(z) / P_{s}^{2}(z)=k^{2}$ and $\psi^{2}=0.0087 \ll 1 \quad\left(\psi=5.3^{\circ}\right)$ conditions. The internal volume of the container is $3.52 \mathrm{ml}$ and it is filled with $3.35 \pm 0.01 \mathrm{ml}$ of $1 \mathrm{cs} \operatorname{PDMS}\left(\rho=816 \mathrm{~kg} \mathrm{~m}^{-3}\right.$, $\sigma=0.0174 \mathrm{~N} \mathrm{~m}^{-1}, \mu=0.000816 \mathrm{~kg} \mathrm{~m}^{-1} \mathrm{~s}^{-1}$, and $\theta=0$ at $20^{\circ} \mathrm{C}$ ) and sealed at 
(a)

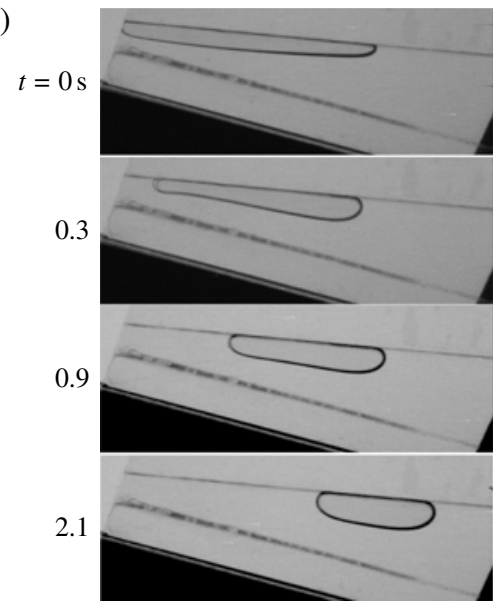

(b)

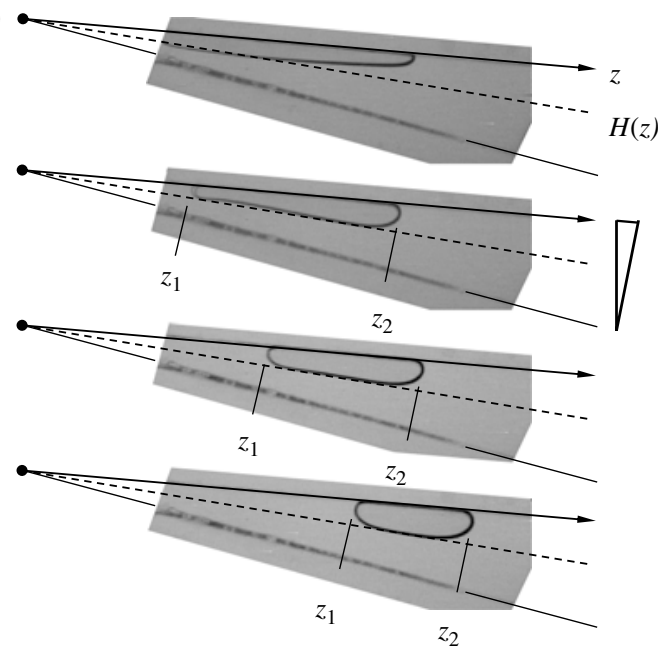

FIGURE 12. (a) Image sequence of a drop tower experiment using the test cell of figure 11, $1 \mathrm{cs}$ PDMS. The container is tilted to force the initial normal gravity interface to nearly replicate the initial weightless state. (b) Corresponding $z$-axis and $H(z)$ shown to demonstrate locations where $z_{1}$ and $z_{2}$ are measured. The interface at $t=0.9 \mathrm{~s}$ shows the greatest degree of edge flow interface curvature and supports the assumption $h\left(z_{1}\right) \approx H\left(z_{1}\right)$ in the most quantitative way despite the presence of finite though diminishing inertia.

the ends with adhesive tape. For this system, $\beta=0.339 \ll 1$ does not necessarily hold. Additionally, the condition $V_{b} / V_{u} \ll 1$ required to ignore gas and liquid volumes in the bulk meniscus regions also does not necessarily hold. Nonetheless, drop tower tests are performed using the container as shown in figure 12. The orientation of the container is chosen such that the initial interface configuration is close to the weightless configuration, reducing the formation time of the capillary-dominated flow, and also so that the most significant flow along the $10^{\circ}$ edge may be viewed with minimal optical distortion. Following the drop, the bulk menisci are established and the capillary-pressure-gradient-driven edge flows displace the bubble from left to right. By the end of the $2.2 \mathrm{~s}$ drop, the bubble has become more spherical, eventually violating the foundational slender edge flow condition $\epsilon^{2} \ll 1$.

The computed known height location $H(z)$ of (3.1) for the $10^{\circ}$ edge is drawn on the container in all of the cut-away images in figure 12(b) using a dashed line. The location of the bulk meniscus 'centreline' is where $z_{1}$ and $z_{2}$ are measured. These quantities are scaled accordingly and plotted with the predictions of (5.12) in figure 13. Despite suspect constraint violations by the end of the $2.2 \mathrm{~s}$ drop where $\beta=0.339 \ll 1, \epsilon^{2} \approx 0.64 \ll 1$, and $V_{b} / V_{u} \approx 0.3 \ll 1$, the asymptotic solutions capture well the general behaviour of the bubble position with under-predictions of $<10 \%$. As discussed in context of figure 3, many applications arise where these constraints are not in question and improved agreement might be expected. In fact, preliminary agreement with large-length-scale long duration experiments performed on the International Space Station (ISS) has also been demonstrated by Jenson et al. (2009) and Blackmore et al. (2011). As shown by others (i.e. Mumley, Radke \& Williams 1986), the ISS experiments also show how pre-existing liquid films on the container surfaces and edges yield faster flows. Depending on the natural capillary drain rate and drain time, up to $25 \%$ increases in flow velocities are observed. This 


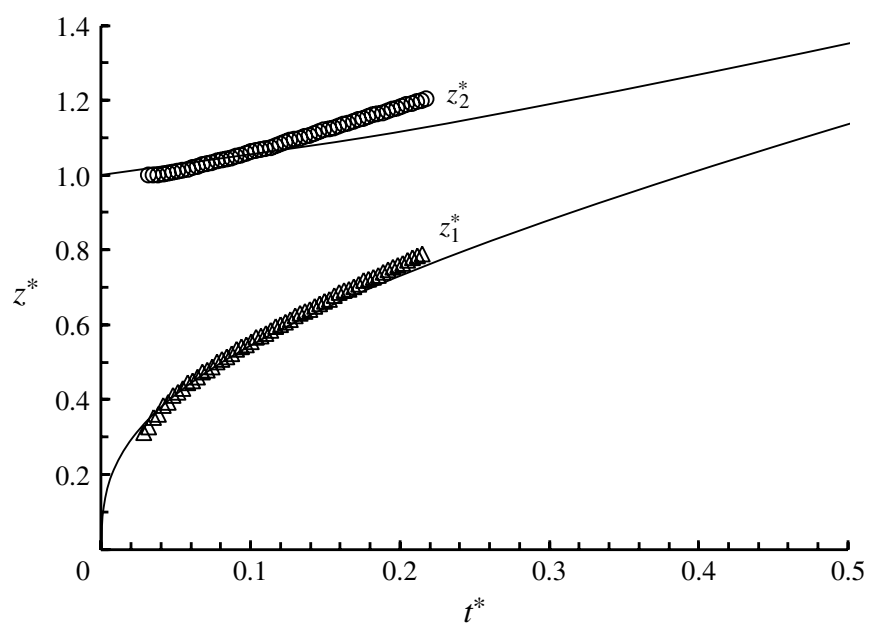

FIGURE 13. Comparison of drop tower experiment data (symbols) from figure 12 for $z_{1}^{*}$ and $z_{2}^{*}$ with quasi-steady predictions (lines) of (5.12).

is well within the range of the predictions compared in figure 13 and may help to account for some of the observed discrepancy.

\section{General dimensional $O(1)$ system}

Sections 4.2 and 5.2 provide a fairly general development of the governing equations for solutions in terms of $\beta$. Though higher-order closed-form analytic solutions may be rare for both drainage and bubble displacement problems, $O(1)$ solutions are possible for many variants of these two flow types. In this section a single $O(1)$ dimensional system of equations is presented for both passive and weakly forced flows. Many additional solutions are possible.

The $O(1)$ solutions derive from the dimensional $O(1)$ jth edge flow equation

$$
0=\frac{\partial}{\partial z}\left(h_{j}^{2} \frac{\partial h_{j}}{\partial z}\right)
$$

subject to $h_{j}\left(z_{1}\right)=H_{1 j}\left(z_{1}\right)$ and $h_{2 j}\left(z_{2}\right)=H_{2 j}\left(z_{2}\right)$. Solution of this equation yields

$$
Q_{j}=\frac{\mathscr{W}_{j} F_{A j}}{3} \frac{H_{2 j}^{3}-H_{1 j}^{3}}{z_{2}-z_{1}},
$$

where $\mathscr{W}_{j}=\sigma\left\langle\bar{w}^{*}\right\rangle_{j} \sin ^{2} \alpha_{j} / \mu f_{j}$ and $H_{j}=R / f_{j}$ with $R(z)$ determined using (4.27). The $O(1)$ flow rate balances at each bulk meniscus are

$$
\begin{aligned}
& z_{1}: \quad A_{s 1} \frac{\mathrm{d} z_{1}}{\mathrm{~d} t}=\sum_{j=1}^{m} Q_{j}+\mathscr{Q}_{1}, \\
& z_{2}: \quad A_{s 2} \frac{\mathrm{d} z_{2}}{\mathrm{~d} t}=\sum_{j=1}^{m} Q_{j}-\mathscr{Q}_{2},
\end{aligned}
$$


and the $O(1)$ bubble volume balance is

$$
\int_{z_{1}}^{z_{2}} A_{s} \mathrm{~d} z=V_{u}(0)-\int_{0}^{t}\left(\mathscr{Q}_{2}+\mathscr{Q}_{1}\right) \mathrm{d} t .
$$

Imposed flows $\mathscr{Q}_{1} \equiv \mathscr{Q}_{1}\left(z_{1}\right)$ and $\mathscr{Q}_{2} \equiv \mathscr{Q}_{2}\left(z_{2}\right)$ are sketched in figure 5 and serve to model additional forced or otherwise externally imposed draining or filling flows. Fluid is added when $\mathscr{Q}_{i}>0$ and removed when $\mathscr{Q}_{i}<0$. In this light, for example, the draining problem of $\S 2.2$ has a negative forced flow (a sink) at the fixed point $z_{1}=0$, where $\mathscr{Q}_{1}=Q\left(z_{1}\right)<0$ and $\mathscr{Q}_{2}=0$. The forced flow solutions hold provided the imposed flow rates (1) are equal to or less than the total edge flow rate $\mathscr{Q}_{i} / Q_{s}$, (2) do not lead to large $C a_{i}$ at moving bulk menisci leading to dynamic contact angle effects with adverse impacts to $H_{i}\left(z_{i}\right)$, and (3) do not violate the incompressible bubble assumption. One of the equations (6.3)-(6.5) is normally redundant. The $O(1)$ system of (6.2)-(6.5) applies for all narrow tapers provided $\psi^{2} \ll 1$ and for numerous arbitrary sections with $m$ wetted edges provided $\sum_{j=1}^{m} \beta_{j} \ll 1$.

\subsection{Constant-area draining with imposed flow}

A sketch of the constant-area draining flow model was provided in figure $5(b)$ for the constant-area $m=1$ container, and where $\mathscr{Q}_{1}<0$ represents the imposed fluid removal rate at $z=0$. The condition $\mathscr{Q}_{2} \gtrless 0$ accounts for the imposed addition or removal of liquid downstream at some location $z>z_{2}$. For $m$ arbitrarily wetted edges, for such flows $H_{1 j}(=0), A_{s 1}\left(=A_{s 2}\right)$, and $H_{2 j}$ are constants. Combining (6.2)-(6.4) and non-dimensionalizing by slightly modified scales

$$
Q \sim \sum_{j=1}^{m} \mathscr{W}_{j} F_{A j} H_{L j}^{* 3} / 3 L \equiv C_{m}, \quad t \sim \frac{A_{s L} L}{C_{m}} \equiv \tau_{s},
$$

produces the simple governing equation

$$
\frac{\mathrm{d} z_{2}^{*}}{\mathrm{~d} \tau^{*}}=\frac{1}{z_{2}^{*}}-\mathscr{Q}_{2}^{*}
$$

where $\tau^{*}=t^{*} / 3$, and $\mathscr{Q}_{2}^{*}=\mathscr{Q}_{2} / C_{m}$ represents an imposed bulk meniscus velocity (or flow rate) at or beyond $z_{2}^{*}$. For these flows (6.5) is satisfied, but is redundant with (6.4). It can be shown that the quasi-steady approach will continue to apply provided $\mathscr{Q}_{2}^{*} \lesssim O(1)$. For large $z_{2}^{*}$ the bulk flow time scale rivals the corner flow time scale, violating the quasi-steady assumption. Fluid is removed when $\mathscr{Q}_{2}^{*}<0$ and added when $\mathscr{Q}_{2}^{*}>0$. A useful variety of analytic solutions is possible for (6.7) depending on the nature of $\mathscr{Q}_{2}^{*}$. Simple example solutions are provided below for cases where $\mathscr{Q}_{2}^{*}=$ const. $= \pm 1$ and $\mathscr{Q}_{2}^{*}=\mathscr{Q}_{2}^{*}\left(z_{2}^{*}\right)$, such that (6.7) is separable and may be re-written for implicit solutions

$$
\tau^{*}=\int \frac{z_{2}^{*}}{1-\mathscr{Q}_{2}^{*} z_{2}^{*}} \mathrm{~d} z_{2}^{*} .
$$

Assuming $\mathscr{Q}_{2}^{*}=-1$ with initial condition $z_{2}^{*}(0)=0$, an assisted draining flow solution to (6.7) yields

$$
\tau^{*}=\ln \left(1+z_{2}^{*}\right)^{-1}+z_{2}^{*},
$$

constrained by $0 \leqslant z_{2}^{*} \lesssim O(1)$, and shown in figure $14(a)$. 
(a)

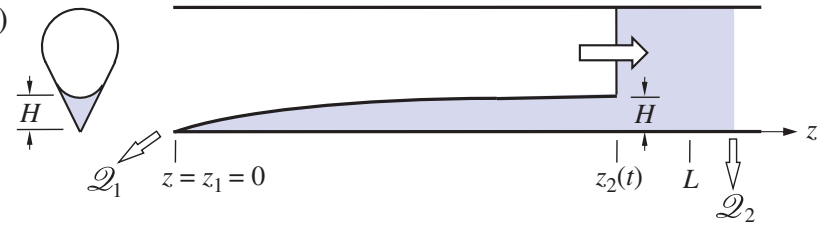

(b)

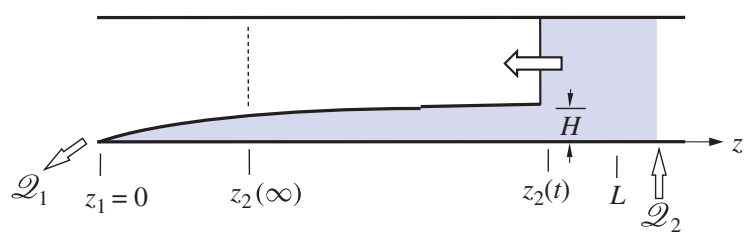

(c)

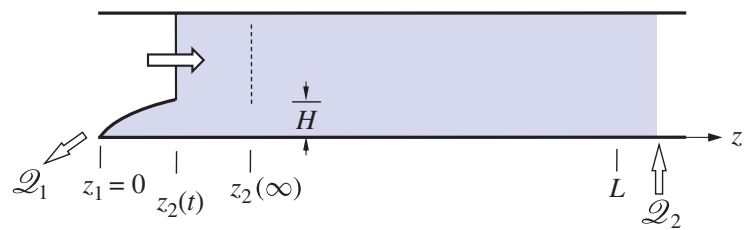

FIGURE 14. (Colour online) Constant-area draining in an $m=1$ section with imposed flow $\mathscr{Q}_{2}$ at $z>z_{2}:(a)$ assisted draining where $\mathscr{Q}_{2}<0(6.9),(b)$ assisted filling with $\mathscr{Q}_{2}>0$ and $z_{2}(t)>z_{2}(\infty)$ (6.10), and $(c)$ assisted filling with $\mathscr{Q}_{2}>0$ and $z_{2}(t)<z_{2}(\infty)(6.10)$.

The case of assisted filling yields two solutions when $\mathscr{Q}_{2}^{*}=1$ that depend on the initial condition. In this situation, as $\tau^{*} \rightarrow \infty,(6.7)$ admits the steady solution $z_{2}^{*}=1$. For initial conditions $z_{2}^{*}(0)<1$, the bulk meniscus recedes from left to right until the edge flow interface slope reduces to the point where the edge flows balance the forced filling flow (see figure 14b,c). Conversely, for initial conditions $z_{2}^{*}(0)>1$, the bulk meniscus advances from right to left until the same balance occurs. Thus, for constant forced filling cases, (6.8) yields solutions

$$
\tau^{*}=\ln \left( \pm 1 \mp z_{2}^{*}\right)^{-1}-z_{2}^{*}
$$

where the upper sign is for $0 \leqslant z_{2}^{*}<1$ and the lower sign is for $1<z_{2}^{*} \lesssim O(1)$. The condition $\mathscr{Q}_{2}^{*}=0$ recovers previous unforced solutions.

If such containers are 'drained' by imposed diffusive capillary flows at some location $z_{\text {cap }}^{*}>z_{2}^{*}$, an appropriate model for this flow might be $\mathscr{Q}_{2}^{*}=-\phi / z_{2}^{*}$ with explicit solution from (6.8) in terms of $t^{*}$

$$
z_{2}^{*}=\left(\frac{2}{3}(1+\phi) t^{*}\right)^{1 / 2}
$$

which recovers (4.41) when $\phi=0$. Such flows may arise in situations where fluid is drained at some location $z^{*}>z_{2}^{*}$ by a capillary flow of smaller pore size (i.e. a porous material), see sketch in figure $15(a)$.

If such containers are 'filled' by an imposed capillary flow arising from the bulk meniscus curvature at $z>z_{2}^{*}$, an appropriate model for this flow might be $\mathscr{Q}_{2}^{*}=1 /\left(1-\phi z_{2}^{*}\right)$ as sketched in figure $15(b)$ with solutions from (6.8) yielding

$$
\tau^{*}=\frac{\ln \left( \pm 1 \mp z_{2}^{*}(1+\phi)\right)^{-1}}{(1+\phi)^{3}}-\frac{z_{2}^{*}}{(1+\phi)^{2}}+\frac{\phi z_{2}^{* 2}}{2(1+\phi)},
$$


(a)

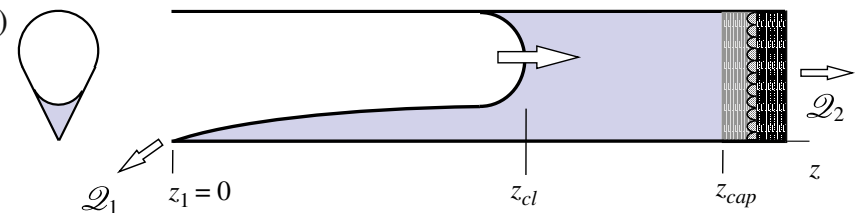

(b)

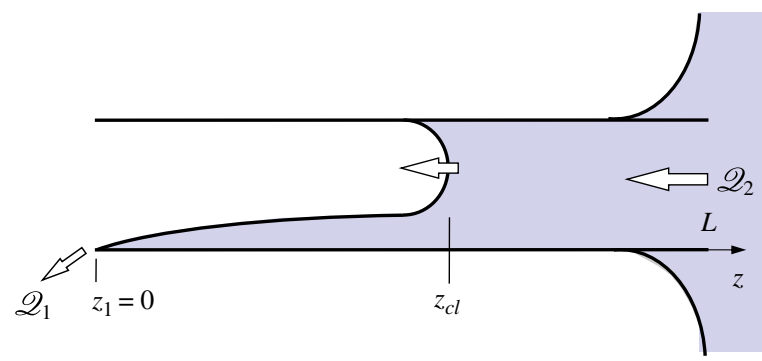

FIgURE 15. (Colour online) Constant-area draining in an $m=1$ section with imposed flows $\mathscr{Q}_{2}$ at $z>z_{2}:(a)$ imposed flow due to diffusive capillary outflow through, say, a porous material with $\mathscr{Q}_{2}<0$ and $(b)$ assisted filling due to bulk capillary rise or inflow with $\mathscr{Q}_{2}>0$.

where $0 \leqslant z_{2}^{*} \lesssim 1 /(1+\phi)$ for the upper sign, and $1 /(1+\phi) \leqslant z_{2}^{*} \lesssim O(1)$ for the lower sign. For all of these combined capillary and imposed flows in arbitrary- $m$ wetted edge sections, the dimensional flow rate removed at $z_{1}=0$ is $\mathscr{Q}_{1}=-\mathscr{Q}_{2}=-C_{m} / z_{2}^{*}$, with, for example, $z_{2}^{*}\left(\tau^{*}\right)$ determined from (6.9)-(6.12).

These $O(1)$ capillary draining flow solutions may be further generalized to allow $H_{1} / H_{2} \equiv b$ for constant-area conduits where $0 \leqslant b \leqslant 1$. The solution approach is readily extended to tapered containers. Simple numerical solutions are possible for arbitrary values of $\mathscr{Q}_{2}$.

\subsection{Bubble displacement with imposed flows: steady and bypass flows}

Equations (6.2)-(6.5) are readily applied to bubble displacement flows with the addition of imposed flows. For example, a steady imposed inflow and outflow such that $\mathscr{Q}_{1}=-\mathscr{Q}_{2}$ results in the unique situation of a stationary bubble sketched in figure 16(a). In this case $\mathrm{d} z_{1} / \mathrm{d} t=\mathrm{d} z_{2} / \mathrm{d} t=0$ and the integral on the right-hand side of (6.5) is identically zero. For the specific case of power law tapers where $H_{j}^{*}=z^{* n}$ and $A_{s}^{*}=z^{* 2 n}$, defining the fixed flow rate $\mathscr{Q}_{2}=-\mathscr{Q}_{1} \equiv \mathscr{Q}$, combining (6.2) and (6.5) yields

$$
\mathscr{Q}=\sum_{j=1}^{m} \frac{\mathscr{W}_{j} F_{A j}}{3}\left(\frac{H_{2 j}^{3}-H_{1 j}^{3}}{z_{2}-z_{1}}\right) .
$$

Non-dimensionalizing this equation we find that because $H_{j}^{*}=z^{* n}$, the term in parentheses on the right-hand side of (6.13) may be pulled out of the summation such that

$$
\mathscr{Q}^{*}=\frac{\mathscr{Q}}{C_{m}}=\frac{z_{2}^{* 3 n}-z_{1}^{* 3 n}}{z_{2}^{*}-z_{1}^{*}} .
$$

This form is valid for arbitrary- $m$ wetted edges. For $L=V_{u}(2 n+1) / A_{s L}$, (6.5) may be applied such that

$$
\mathscr{Q}^{*}=\frac{\left(1+z_{1}^{* 2 n+1}\right)^{3 n /(2 n+1)}-z_{1}^{* 3 n}}{\left(1+z_{1}^{* 2 n+1}\right)^{1 /(2 n+1)}-z_{1}^{*}} .
$$


(a)

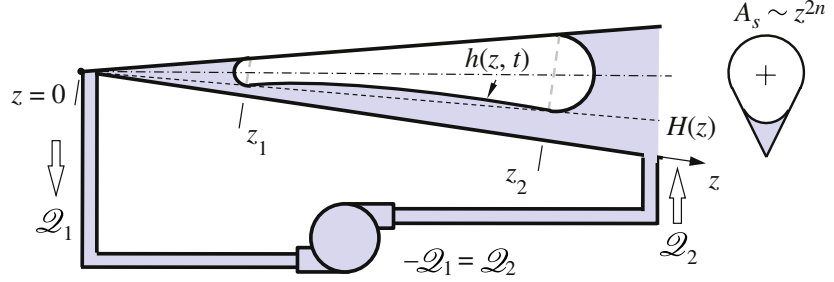

(b)

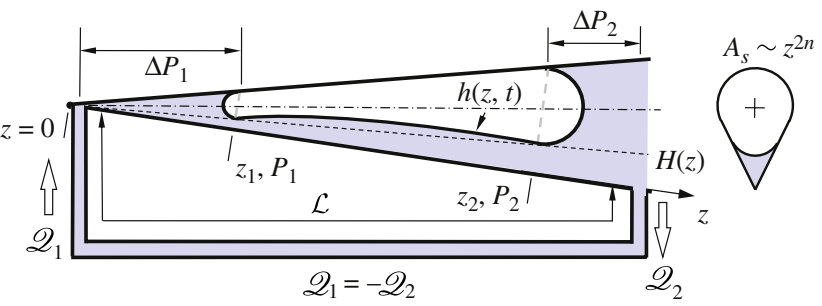

FIGURE 16. (Colour online) Bubble displacement with bypass flows: (a) conditions for stationary bubble, $(b)$ parallel flow capillary bypass conduit.

Many solutions are possible, but for the case $n=1, A_{s}^{*}=z^{* 2}, H^{*}=z^{*}$, and (6.15) yields

$$
z_{1}^{*}=\frac{3^{1 / 2}\left(4 \mathscr{Q}^{* 3}-1\right)^{1 / 2}-3}{6 \mathscr{Q}^{*}}, \quad z_{2}^{*}=\left(1+z_{1}^{* 3}\right)^{1 / 3}=\frac{3^{1 / 2}\left(4 \mathscr{Q}^{* 3}-1\right)^{1 / 2}+3}{6 \mathscr{Q}^{*}},
$$

valid for $\mathscr{Q}^{*} \geqslant 1$ and all arbitrary- $m$ wetted containers.

A second example of bubble displacement with imposed flow is sketched in figure 16(b), where a 'bypass' conduit provides a second path for fluid to move from the broader region of the tapered section to the narrower region. If the pressure losses in the tapered section are small (namely, $\left|\Delta P_{1}\right|+\left|\Delta P_{2}\right| \ll P_{2}-P_{1}$, see figure $16 b$ ), the bypass flow may be modelled using the difference in capillary curvatures at $z_{1}$ and $z_{2}$ as the driving force with only viscous resistance in the bypass conduit. As in the previous example, $\mathscr{Q}_{1}=-\mathscr{Q}_{2}$ for this flow which simplifies (6.5) and renders (6.4) redundant. Using the scaling of (6.6), for power law tapers it can be shown that

$$
(1+B) z_{1}^{* 2 n} \frac{\mathrm{d} z_{1}^{*}}{\mathrm{~d} \hat{\tau}^{*}}=\frac{z_{2}^{* 3 n}-z_{1}^{* 3 n}}{z_{2}^{*}-z_{1}^{*}}+B\left(\frac{1}{z_{1}^{*}}-\frac{1}{z_{2}^{*}}\right),
$$

subject to $z_{1}^{*}(0)=0$, where $\hat{\tau}^{*}=\tau^{*} /(1+B)$ and the bypass parameter $B$ serves as a ratio of the initial bypass to total edge flow rate. For a single circular bypass tube of radius $r$ and length $\mathscr{L}$, for example, $B=\pi \sigma r^{4} / 8 \mathscr{L}\left(H_{L j} f_{j}\right) C_{m}$, where $H_{L j} f_{j}=$ const. $=R(z=L)$. Equation (6.17) may be written in implicit form

$$
\hat{\tau}^{*}=(1+B) \int \frac{z_{1}^{* 3 n} z_{2}^{* n}\left(z_{2}^{*}-z_{1}^{*}\right)}{z_{1}^{* n} z_{2}^{* n}\left(z_{2}^{* 3 n}-z^{* 3 n}\right)+B\left(z_{2}^{* n}-z_{1}^{* n}\right)^{2}} \mathrm{~d} z_{1}^{*}
$$

with $z_{2}^{*}=\left(1+z_{1}^{* 2 n+1}\right)^{1 /(2 n+1)}$ and may be integrated for various values of $n$ and $B$. Solutions for $B \ll 1, B=1$, and $B \gg 1$ for $n=1$ are listed in the Appendix.

\subsection{Bubble displacement in stepped containers}

Piecewise tapered or 'stepped' containers are sketched in figure $9(d-e)$. The stepped $m=1$ section of figure $9(d)$ is annotated in figure $10(d)$, but any arbitrary- $m$ wetted 


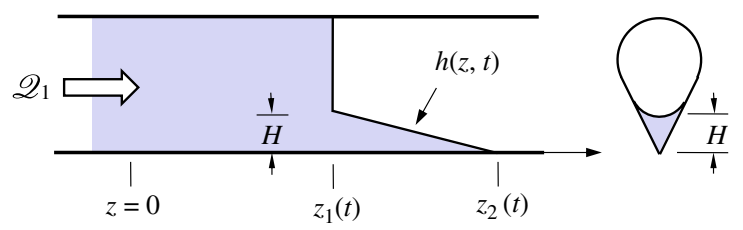

FIGURE 17. (Colour online) Steady weakly forced flow with advancing edge flow.

edge containers conform to the analysis. In figure $10(d), z_{1}(0)=0, z_{2}(0)=L$ and the flow terminates when $z_{1}=L$. For $m$-arbitrary geometries the dimensional boundary conditions for the edge flow (6.1) are constants: $h_{j}\left(z_{1}\right)=H_{1 j}$ and $h_{j}\left(z_{2}\right)=H_{2 j}$ and $b \equiv H_{1 j} / H_{2 j}$. The fixed ratio $b$ is independent of $j$ and may be expressed in terms of $\beta$. The non-dimensionalized form of (6.3) and the integrated form of (6.5) are

$$
\frac{\mathrm{d} z_{1}^{*}}{\mathrm{~d} \tau^{*}}=\frac{1-b^{3}}{z_{2}^{*}-z_{1}^{*}}
$$

and

$$
z_{2}^{*}=1+b^{2} z_{1}^{*},
$$

respectively. Substitution of (6.20) into (6.19) produces

$$
z_{1}^{*}=\frac{1-\left(1-2\left(1-b^{2}-b^{3}+b^{5}\right) \tau^{*}\right)^{1 / 2}}{1-b^{2}},
$$

where the flow ceases abruptly when $\tau^{*}=(1 / 2)\left(1-b^{2}-b^{3}+b^{5}\right)^{-1}$. The dimensionless flow rate across the bubble is

$$
\mathscr{Q}^{*}=\frac{1-b^{3}}{\left(1-2\left(1-b^{2}-b^{3}+b^{5}\right) \tau^{*}\right)^{1 / 2}} .
$$

The addition of forced flows $\mathscr{Q}_{1}^{*}$ and $\mathscr{Q}_{2}^{*}$ is straightforward.

\section{Special case of a steady advancing bulk meniscus with edge flows}

The historic problem of imbibition in infinite containers with interior edges studied by Dong \& Chatzis (1995), Romero \& Yost (1996), and Weislogel \& Lichter (1998) does not lend itself to the quasi-steady approach. This is because the edge flow and bulk meniscus time scales are always the same. However, the current success of the dynamical boundary condition applied at the bulk meniscus $h\left(z_{i}, t\right)=H\left(z_{i}\right)$ suggests that other forced flows might be investigated provided they are steady. For example, for a uniform $m=1$ section sketched in figure 17, fluid is forced left to right at a steady flow rate $\mathscr{Q}_{1}$. Provided capillary forces continue to dominate the bulk meniscus curvature $\left(\mathrm{Ca}_{b} \ll 1\right)$, one should be able to apply $h_{j}\left(z_{1}\right)=H_{1 j}$ determined by the de Lazzer et al. method.

Invoking local length scale $L_{j}$ at the $j$ th of $m$ wetted edges, the dimensionless steady solution to the edge flow equation (4.24) is the linear one,

$$
h_{j}^{*}=\frac{z_{2 j}^{*}-z_{j}^{*}}{z_{2 j}^{*}-z_{1}^{*}},
$$


where conditions $h_{j}^{*}\left(z_{1}^{*}\right)=1$ and $h^{*}\left(z_{2 j}^{*}\right)=0$ are satisfied. Thus,

$$
h_{j}^{* 2} \frac{\partial h_{j}^{*}}{\partial z_{j}^{*}}=-\frac{1}{z_{2 j}^{*}-z_{1}^{*}},
$$

where $z_{1 j}^{*}=z_{1}^{*}$ is applied. A dimensional bulk meniscus flow rate balance may be applied at $z_{1}$ with the addition of the imposed steady flow rate

$$
\mathscr{Q}_{1}+\left.\sum_{j=1}^{m} F_{A j} \mathscr{W}_{j}\left(h_{j}^{2} \frac{\partial h_{j}}{\partial z}\right)\right|_{z_{1}}=\left(A_{s}-\sum_{j=1}^{m} F_{A j} H_{1 j}^{2}\right) \frac{\mathrm{d} z_{1}}{\mathrm{~d} t} .
$$

Choosing scales $z_{j} \sim L_{j} \equiv \mathscr{W}_{j} H_{1 j} A_{s} / \mathscr{Q}_{1}, z_{1} \sim L$, and $t \sim A_{s} L / \mathscr{Q}_{1}$, and employing (7.2), (7.3) becomes

$$
1-\sum_{j=1}^{m} F_{A j} H_{1 j}^{* 2}\left(\frac{1}{z_{2 j}^{*}-z_{1}^{*}}\right)=\left(1-\sum_{j=1}^{m} \beta_{j}\right) \frac{\mathrm{d} z_{1}^{*}}{\mathrm{~d} t^{*}} .
$$

But the flow is steady, $\mathrm{d} z_{1}^{*} / \mathrm{d} t^{*}=1$ and $z_{2 j}^{*}-z_{1}^{*}=$ const. Thus, with the definition $\beta_{j}=F_{A j} H_{1 j}^{* 2}$, inspection of (7.4) reveals

$$
z_{2 j}^{*}-z_{1}^{*}=1 .
$$

Clearly, the velocity of the bulk meniscus is equal to the velocity of the advancing tip. Thus, the solution is one of an additive separation of variables and if a $j$-dependent time scale can be defined by $t_{j} \sim \mathscr{W}_{j} H_{1 j} A_{s}^{2} / \mathscr{Q}_{1}^{2}$, one finds for each edge flow that

$$
h_{j}^{*}=1+t_{j}^{*}-z_{j}^{*} .
$$

In dimensional terms

$$
z_{1}=z_{1 j}=\mathscr{Q}_{1} / A_{s}, \quad z_{2 j}=z_{1}+\mathscr{W}_{j} H_{1 j} A_{s} / \mathscr{Q}_{1} .
$$

The edge flows are always contained within $z_{1} \leqslant z_{j} \leqslant z_{2 j}$, and the length of the edge flow increases with decreasing $\mathscr{Q}_{1} / A_{s}$.

The form of this solution was presented by Weislogel (1996) as a self-similar 'linear tip' solution because it was found to be the only exact solution for all orders of $\beta$ for advancing edge flows that accounted for interfacial curvature in both cross-flow and streamwise directions, the latter being identically zero in this case. However, the application of that solution was understood not to apply to the current problem. For small $\mathscr{Q}_{1} / A_{s}$, long linear interface profiles are predicted which could be observed experimentally. No such observations have occurred to our knowledge.

\section{Sample applications}

The solutions obtained herein are readily dimensionalized for applications. Two quick examples of flow rate optimizations are provided here for the draining similarity solution of (4.17)-(4.21) and the exact power law bubble displacement solution of (5.6)-(5.7). These solutions are chosen because they provide optimal container dimensions that are not restricted by the low saturation limit, $\beta \ll 1$.

The constant-area $m$-regular draining flow similarity solution of $\S 4.2$ may be redimensionalized as follows for bulk meniscus location $z_{2}$ and drain rate $Q_{1}$ for sections where either area $A_{s}$ or perimeter $P_{s}$ are held constant: 
(I) in $A_{s}$-fixed form

$$
\begin{gathered}
z_{2}=\left(\frac{2 \sigma A_{s}^{1 / 2} t}{3 \mu}\right)^{1 / 2} k^{-1 / 2}\left(\beta F_{\mathscr{W}} F_{H}\right)^{1 / 2} \eta_{2}, \\
Q=\left.\left(\frac{3 \sigma A_{s}^{5 / 2}}{2 \mu t}\right)^{1 / 2} k^{-1 / 2}\left(\beta F_{\mathscr{W}} F_{H}\right)^{1 / 2}\left(F^{2} F^{\prime}\right)\right|_{0}
\end{gathered}
$$

(II) in $P_{s}$-fixed form

$$
\begin{gathered}
z_{2}=\left(\frac{2 \sigma P_{s} t}{3 \mu}\right)^{1 / 2}\left(\beta F_{\mathscr{W}} F_{H}\right)^{1 / 2} \eta_{2}, \\
Q=\left.\left(\frac{3 \sigma P_{s}^{5}}{2 \mu t}\right)^{1 / 2} k^{2}\left(\beta F_{\mathscr{W}} F_{H}\right)^{1 / 2}\left(F^{2} F^{\prime}\right)\right|_{0},
\end{gathered}
$$

where $\beta=m \beta_{j}$ and $F(\eta)$ and $\eta_{2}$ are given by (4.17) and (4.18), respectively, and $F_{\mathscr{W}} \equiv\langle\bar{w}\rangle{ }^{*} \sin ^{2} \alpha / f$ and $F_{H} \equiv F_{H P} / f$ (see (4.32)). The first term in parentheses on the right-hand side of these relationships is independent of geometry. The remaining terms include the dimensionless area-perimeter ratio $k^{2}$ and the geometric term $\beta F_{\mathscr{W}} F_{H}$. The coefficients $\eta_{2}$ and $\left.\left(F^{2} F^{\prime}\right)\right|_{0}$ are functions of $\beta$ only. Important quantities such as optimal flow rates for example are readily determined by computing these geometric components for a variety of conduit types. For example, for the $m=1$ section discussed throughout this work, for perfectly wetting fluids, it can be shown that $4 k^{2}=(\pi / 2+\alpha+\cot \alpha)^{-1}$ and that the flow rate is maximized at $\alpha \approx 2.2^{\circ}$ when comparing fixed-area sections $\left(A_{s}\right.$-fixed, $P_{s}$ varies, (8.2)). For fixed-perimeter sections $\left(P_{s}\right.$-fixed, $A_{s}$ varies, (8.4)) the optimal edge half-angle is $\alpha \approx 15^{\circ}$. Such angles are found to be weakly dependent on $\beta$ which suggests that the $O(1)$ solutions may be adequate to guide optimal designs, even when $\beta \ll 1$ is not necessarily satisfied. It is also interesting to note that variations in computed flow rates between $O(1)$ - and $O\left(\beta^{4}\right)$-accurate solutions differ by less than $12 \%$, even for this geometry where $\beta$ approaches 1 . Variations in $z_{2}$ are less than $2.5 \%$.

A similar exercise for the exact steady bubble displacement solution of (5.6) and (5.7) $\left(A_{s} \sim z^{2 / 3}\right)$ yields dimensional forms under the definitions $A_{s}=\left(A_{o} z\right)^{2 / 3}$ and $P_{o}=A_{o}^{1 / 2} / k$ :

(III) for $A_{o}$ and $V_{u}$ fixed

$$
\begin{gathered}
z_{1}=\left(\frac{5}{9} \frac{\sigma}{\mu} A_{o}^{1 / 3} t\right)^{3 / 5}\left(\frac{\beta}{1-\beta}\right)^{3 / 5} k^{-3 / 5}\left(F_{\mathscr{W}} F_{H}\right)^{3 / 5} \\
z_{2}=\left(\frac{5}{3} \frac{V_{u}}{A_{o}^{2 / 3}}\right)^{3 / 5}\left(\frac{1}{1-\beta}\right)^{3 / 5}\left[1+\left(\frac{1}{3} \frac{\sigma}{\mu} \frac{A_{o}}{V_{u}} t\right) k^{-1}\left(\beta F_{\mathscr{W}} F_{H}\right)\right]^{3 / 5}, \\
Q=\left(\frac{\sigma}{\mu} A_{o}\right) k^{-1}\left(\beta F_{\mathscr{W}} F_{H}\right) ;
\end{gathered}
$$

(IV) for $P_{o}$ and $V_{u}$ fixed

$$
\begin{gathered}
z_{1}=\left(\frac{5}{9} \frac{\sigma}{\mu} P_{o}^{2 / 3} t\right)^{3 / 5}\left(\frac{\beta}{1-\beta}\right)^{3 / 5} \frac{1}{k^{1 / 5}}\left(F_{\mathscr{W}} F_{H}\right)^{3 / 5}, \\
z_{2}=\left(\frac{5}{3} \frac{V_{u}}{P_{o}^{4 / 3}}\right)^{3 / 5}\left(\frac{1}{1-\beta}\right)^{3 / 5} \frac{1}{k^{4 / 5}}\left[1+\left(\frac{1}{3} \frac{\sigma}{\mu} \frac{P_{o}^{2}}{V_{u}} t\right) k\left(\beta F_{\mathscr{W}} F_{H}\right)\right]^{3 / 5},
\end{gathered}
$$




$$
Q=\left(\frac{\sigma}{\mu} P_{o}^{2}\right) k\left(\beta F_{\mathscr{W}} F_{H}\right) .
$$

These forms are again arranged in part to separate non-geometric and geometric quantities, and from (8.7) and (8.10), maximal flow rates in tapered $m=1$ sections with perfect wetting fluids are computed to be $\alpha \approx 1.6^{\circ}$ and $\alpha \approx 7.5^{\circ}$ for fixed-area and fixed-perimeter sections, respectively.

\section{Summary}

In this work, assumed known height boundary conditions are applied to bulk menisci that permit closed-form analytic solutions for a variety of important flows driven passively by the interior edges of containers. For low-saturation flows, these assumptions allow quasi-steady solutions for combined capillary and weakly forced flows for constant and slightly varying duct sections. Such flows may be exploited in numerous capillary fluidics applications requiring passive fluid control, transport, and phase separations. The solutions capture the impact of geometry permitting efficient and improved design and analysis. Despite qualitative agreement with simple experiments reported herein, a thorough experimental investigation of the validity of the approach is yet to be conducted. Numerical experiments employing benchmarked codes along these lines would do well. Nonetheless, the successful demonstration of the analysis identifies a path along which to pursue a broad range of phenomena, including flows with other motive forces, phase-change heat transfer, property variation, sources, bubble compressibility, and increasingly complex geometries.

The present analysis is restricted to parallel liquid flows along slender containers or conduits with fixed edge angles. The bubble is treated as a passive incompressible gas. In regions of the containers where edge flows are present, the liquid content is low, while the bubble volume 'contained' within the bulk menisci is neglected. All velocities normal to the contact line are assumed small such that the static contact angle may be applied everywhere. Closed form implicit and explicit zeroth-order solutions are provided except in special cases where exact or higher-order explicit forms are easy.

Most of the quasi-steady flows addressed herein can be demonstrated to perform passive phase separations by forcing bubbly flows through the narrow edge flows where the bubbles impinge against the bulk meniscus, coalesce, and in effect, collect and separate the fluid phases into two large continuous bodies (see figure 2). Such low-speed 'multi-phase' flows might also be studied using the approach presented herein while awaiting numerical methods capable of the complete analysis. Certain challenges also remain for the study of flows in tapering sections with vane edges, such as the container types sketched in figure $9(f, g)$. Such geometries are useful to provide a practical passive means for fluid migration, but the tapering vane edge presents a boundary condition difficulty requiring mixed pinned and free contact line conditions. Solutions for related problems are possible (i.e. Romero \& Yost 1996 and Weislogel 2001), but complications with the de Lazzer et al. approach are probable and it is likely that solutions might only arise for an even more restricted set of geometries. Thus, the impact of common complexities such as re-entrant edges and strongly three-dimensional geometries on the extension of the de Lazzer et al. boundary condition remains unaddressed.

Partial wetting systems with finite contact angle hysteresis continue to present challenges for analysis. This is unfortunate because numerous systems requiring the processing of water exhibit partial wettability (i.e. PEM fuel cells and waste water 
recycling aboard spacecraft). In such cases, experiments for specific systems are required to guide further applications of the present analytic approach if quantitative predictions and design guides are to continue to be developed.

This work was supported in part by NASA under contract NNC05AA29A and NSF under grant CTS-0521890. The authors wish to thank astronauts D. Pettit and S. Williams for providing entertaining demonstrations from the International Space Station and NASA Glenn Research Center for access to a drop tower.

\section{Appendix}

\section{A.1. Higher-order asymptotic solutions of (4.18) and (4.19)}

Performing the asymptotic solution method leading to (4.18) and (4.19) to $O\left(\beta^{10}\right)$ yields

$$
\begin{aligned}
\eta_{2}= & +\frac{3 \beta}{8}+\frac{375 \beta^{2}}{1664}+\frac{485 \beta^{3}}{3072}+\frac{15304397 \beta^{4}}{127369216}+\frac{687434933 \beta^{5}}{7132676096} \\
& +\frac{905470046797 \beta^{6}}{11298158936064}+\frac{169692395414747 \beta^{7}}{2480573561962496}+\frac{11051294096453709253 \beta^{8}}{185557726755792355328} \\
& +\frac{23215590878574079168319 \beta^{9}}{440885158771762636259328} \\
& +\frac{76363095190530678051655169 \beta^{10}}{1620105996766637100707610624}+O\left(\beta^{11}\right),
\end{aligned}
$$

and flow rate draining function

$$
\begin{aligned}
\left.\left(F^{2} F^{\prime}\right)\right|_{0}= & \frac{1}{3}-\frac{3 \beta}{40}-\frac{411 \beta^{2}}{41600}-\frac{46331 \beta^{3}}{14976000}-\frac{22263213 \beta^{4}}{15921152000}-\frac{1336964121 \beta^{5}}{1714585600000} \\
& -\frac{261467378713787 \beta^{6}}{529601200128000000}-\frac{19990270464199251 \beta^{7}}{58981028986880000000} \\
& -\frac{1372078934035641723867317 \beta^{8}}{5581228500076566937600000000} \\
& -\frac{671201314439087978340164303 \beta^{9}}{3616636068049615375564800000000} \\
& -\frac{842010066551752503586348683881 \beta^{10}}{5828917545932596476641280000000000}+O\left(\beta^{11}\right) .
\end{aligned}
$$

A.2. Solution to (6.18)

For $n=1,(6.18)$ yields analytic solutions in special cases:

$B \ll 1$ (* dropped):

$$
\hat{\tau}=\frac{1+4 B}{4}\left(z_{2}^{4}-z_{1}^{4}-1\right)-2 B z_{12}^{2} F_{1}\left(\frac{2}{3}, \frac{1}{3} ; \frac{5}{3} ;-z_{1}^{3}\right)+O\left(B^{2}\right),
$$

$B=1\left({ }^{*}\right.$ dropped $):$

$$
\begin{aligned}
\hat{\tau}= & \frac{1}{4}\left(2 z_{1}+z_{1}^{4}+z_{2}-z_{1}^{3} z_{2}-1\right)+\frac{1}{2^{4 / 3} 3^{1 / 2}}\left(\tan ^{-1}\left[\frac{1+2^{4 / 3}}{3^{1 / 2}}\right]\right. \\
& \left.-\tan ^{-1}\left[\frac{1+2^{4 / 3} z_{2}}{3^{1 / 2}}\right]+\tan ^{-1}\left[\frac{1-2^{4 / 3} z_{1}}{3^{1 / 2}}\right]-\frac{\pi}{6}\right)
\end{aligned}
$$




$$
\begin{aligned}
& +\frac{1}{32^{4 / 3}} \ln \left[\frac{1-2^{1 / 3} z_{2}}{\left(1-2^{1 / 3}\right)\left(1+2^{1 / 3} z_{1}\right)}\right] \\
& +\frac{1}{62^{4 / 3}} \ln \left[\frac{\left(1+2^{1 / 3}+2^{1 / 3}\right)\left(1-2^{1 / 3} z_{1}+2^{2 / 3} z_{1}^{2}\right)}{1+2^{1 / 3} z_{2}+2^{2 / 3} z_{2}}\right],
\end{aligned}
$$

$B \gg 1$ (* dropped):

$$
\begin{aligned}
\hat{\tau}= & \frac{1}{28}\left[3+3 z_{1}^{4}+2 z_{1}^{2} z_{2}^{2}-3 z_{2}^{4}+4 z_{1}^{5} z_{2}^{2}+4 z_{1}^{4} z_{2}^{3}+4 z_{1}^{3} z_{2}^{4}-2 z_{1}^{2} F_{1}\left(\frac{2}{3}, \frac{1}{3} ; \frac{5}{3} ;-z_{1}^{3}\right)\right] \\
& +O\left(B^{-1}\right),
\end{aligned}
$$

where ${ }_{2} F_{1}(a, b ; c ; x)$ is the hypergeometric function. It can be shown that the short time limiting solutions are $z_{1}^{*}=\hat{\tau}^{* 1 / 3}$ and $z_{2}^{*}=1+\hat{\tau}^{*} / 3$ and that from (6.17) the large$B$ limit is only maintained provided $B \gg\left(1-z_{1}^{*} / z_{2}^{*}\right)^{-2}$, which becomes increasingly difficult to satisfy as $\hat{\tau}^{*} \rightarrow \infty$ since in this limit $z_{1}^{*} \rightarrow z_{2}^{*}$. Solutions for other $n$ are possible (i.e. $n=1 / 3,1 / 2$, etc.) and different mathematical forms of taper types may be pursued with a similar approach.

\section{REFERENCES}

Akhtara, N., Qureshib, A., ScholtaA, J., Hartniga, C., Messerschmidta, M. \& LEHNERTA, W. 2009 Investigation of water droplet kinetics and optimization of channel geometry for pem fuel cell cathodes. Intl J. Hydrogen Energy 34 (3), 3104-3111.

Ayyaswamy, P. S., Catton, I. \& Edwards, D. K. 1974 Capillary flow in triangular grooves. $J$. Appl. Mech. 41 (4b), 332.

BANKOFF, S. G. 1957 Ebullition from solid surfaces in the absence of a pre-existing gaseous phase. Trans. ASME 79, 735-740.

BiCO, J. \& QUÉRÉ, D. 2002 Rise of liquids and bubble in angular capillary tubes. J. Colloid Interface Sci. 247, 162-166.

Blackmore, W., Weislogel, M. M., Chen, Y., Bunnell, C. T., Kiewidt, L. \& Klatte, J. The Capillary Flow Experiments (CFE-2) aboard the ISS: Status. AIAA Paper 2011-1196, pp. 1-10. 49th AIAA Aerospace Sciences Meeting, Jan. 2011.

Bolleddula, D. 2007 Capillary flow analysis and computation of dewetting and wetting resistances in angular geometries. Master's thesis, Portland State University.

ChAto, D. J. 2008 Cryogenic fluid transfer for exploration. Tech Rep. TM-215286, Lewis Research Center, Cleveland, OH.

Chato, D. J. \& MARTin, T. A. 2006 Vented tank resupply experiment: flight test results. J. Spacecr. Rockets 3, 5 .

Chen, Y., Weislogel, M. M. \& NARdin, C. 2006 Capillary-driven flows along rounded interior corners. J. Fluid Mech. 566, 235-271.

Chernous' KO, F. L. 1968 Introduction to the Dynamics of a Body Containing a Liquid Under Zero-Gravity Conditions. Computing Center of the Academy of Sciences of the USSR (in Russian). Vychislitel'nyi Tsentr Akad. SSSR. The problem of equilibrium of a fluid subject to forces of gravity and surface tension. [translated for author M.M.W. by Lev A. Slobozhanin].

Collicott, S. H. \& Weislogel, M. M. 2004 Computation of capillary instabilities using surface evolver. AIAA J. 42 (2), 289-295.

Collier, J. 1972 Convection Boiling and Condensation, 2nd edn. McGraw Hill.

Concus, P. \& FInN, R. 1969 On the behaviour of a capillary free surface in a wedge. Proc. Natl Acad. Sci. USA 63, 292-299.

Concus, P. \& FInN, R. 1990 Capillary surface in microgravity. In Low-Gravity Fluid Dynamics and Transport Phemomena, Progress in Astronautics and Aeronautics, vol. 130. AIAA.

Concus, P., Finn, R. \& Weislogel, M. 2000 Measurement of critical contact angle in a microgravity experiment. Exp. Fluids 28 (3), 197-205. 
Dong, M. \& Chatzis, I. 1995 The imbibition and flow of a wetting liquid along the corners of a square capillary tube. J. Colloid Interface Sci. 172 (2), 278-288.

FInN, R. \& NeEL, R. W. 1999 C-singular solutions of the capillary problem. J. Reine Angew. Math. 512, 1-25.

GRIFFITH, P. \& WALLIS, J. D. 1960 The role of surface conditions in nucleate boiling. AIChE J. 56, 49-63.

Jenson, R. M., Weislogel, M. M., Tavan, N. T., Chen, Y., Semerjian, B., Bunnell, C. T., Collicott, S. H., Klatte, J. \& Dreyer, M. E. 2009 The capillary flow experiments aboard the international space station: increments 9-15, August 2004-December 2007. Tech Rep. CR-2009-215586, NASA.

KAST, W. 1964 Beteutung der keimbildung und der instationanen wrmebertragung fur den wrmebertraga bei blasenverdampfung und tropfenkondensation. Chemie Ing. Teckn. 36 (9), 933-940.

Khare, K., Brinkmann, M., Bruce, M., Law, B. M., Gurevich, E. L., Herminghaus, S. \& SEEMANN, R. 2007 Dewetting of liquid filaments in wedge-shaped grooves. Langmuir 23, $12138-12141$.

Klatte, J., HaAke, D., Weislogel, M. M. \& Dreyer, M. E. 2008 A fast numerical procedure for steady capillary flow in open capillary channels. Acta Mech. 201, 269-276.

KovsceK, A. R. \& RADKe, C. J. 1996 Gas bubble snap-off under pressure driven flow in constricted non-circular capillaries. Colloids Surf. 117, 56-76.

De Lazzer, A., Langbein, D., Dreyer, M. \& Rath, H. J. 1996 Mean curvature of liquid surfaces in cylindrical containers of arbitrary cross-section. Microgravity Sci. Technol. 9 (3), $208-219$.

Lekan, J., Gotti, D., Jenkins, A., Owens, J. C. \& Johnston, M. R. April 1996 Users guide for the 2.2 second drop tower of the NASA lewis research centre. Tech. Mem. 107090. NASA, Lewis Research Center.

Mumley, T. E., Radke, C. J. \& Williams, M. C. 1986 Kinetics of liquid/liquid capillary rise, Parts i and ii. J. Colloid Interface Sci. 109 (2), 398-425.

Ponomarenko, A., Clanet, C. \& Quéré, D. 2011 Capillary rise in wedges. J. Fluid Mech. 666, $146-154$.

RAMÉ, E. \& Weislogel, M. M. 2009 Gravity effects on capillary flows in sharp corners. Phys. Fluids 21 (4), 042106.

RANSOHOFF, T. C. \& RADKE, C. J. 1988 Laminar flow of a wetting liquid along the corners of a predominantly gas-occupied non-circular pore. J. Colloid Interface Sci. 121 (392).

Romero, L. A. \& Yost, F. G. 1996 Flow in an open channel capillary. J. Fluid Mech. 322, $109-129$.

Rosendahl, U., Ohlhoff, A. \& DReYer, M. E. 2004 Choked flows in open capillary channels: theory, experiment and computations. J. Fluid Mech. 518, 187-214.

Sobhan, C. B., Rag, R. L. \& Peterson, G. P. 2007 A review and comparative study of the investigations on micro heat pipes. Intl J. Energy Res. 31, 664-688.

SUMAN, B. \& KUMAR, P. 2005 An analytical model for fluid flow and heat transfer in a micro-heat pipe of polygonal shape. Intl J. Heat Mass Transfer 48, 4498-4509.

TAVAN, N. 2009 Critical geometric wetting phenomena: study of capillary driven flow in the CFE Vane-Gap experiment aboard the International Space Station. Master's thesis, Portland State University.

WANG, C. X., XU, S. H., Sun, Z. W. \& Hu, W. R. 2010 A study of the influence of initial liquid volume on the capillary flow in an interior corner under microgravity. Intl J. Heat Mass Transfer 53 (9-10), 1801-1807.

Weislogel, M. M. 1996 Capillary flow in an interior corner. PhD thesis, Northwestern University.

Weislogel, M. M. 2001 Capillary flow in containers of polygonal section. AIAA J. 39 (12), 2320-2326.

Weislogel, M. M. 2003 Some analytical tools for fluids management in space: isothermal capillary flows along interior corners. Adv. Space Res. 32 (2), 163-170.

Weislogel, M. M. \& Collicott, S. H. 2004 Capillary rewetting of vaned containers: spacecraft tank rewetting following thrust resettling. AIAA J. 42 (12), 2551-2561. 
Weislogel, M. M. \& Lichter, S. 1998 Capillary flow in an interior corner. J. Fluid Mech. 373, 349-378.

Weislogel, M. M. \& NARdin, C. L. 2005 Capillary driven flow along interior corners formed by planar walls of varying wettability. Microgravity Sci. Technol. 17 (3), 45-55.

Weislogel, M. M., Jenson, R. \& Bolleddula, D. 2007 Capillary driven flows in weakly three-dimensional polygonal containers. AIAA Paper 2007-748, pp. 1-13. 45th AIAA Aerospace Sci. Meeting and Exhibit, Reno, Jan. 2007.

Weislogel, M. M., Chen, Y \& Bolledulla, D 2008 A better non-dimensionalization scheme for slender laminar flows: The Laplacian operator scaling method. Phys. Fluids 20 (2), $163-170$.

Weislogel, M. M., Chen, Y., Collicott, S. H., Bunnell, C. T., Green, R. D. \& Bohman, D. Y. $2009 a$ More handheld fluid interface experiments for the International Space Station (CFE-2). AIAA Paper 2009-0615, pp. 1-10. 47th AIAA Aerospace Sciences Meeting, Orlando,.

Weislogel, M. M., Thomas, E. A. \& Graf, J. C. $2009 b$ A novel device addressing design challenges for passive fluid phase separations aboard spacecraft. Microgravity Sci. Technol. 21 (3), 257-268.

YI, T. \& WONG, H. 2007 Theory of slope-dependent disjoining pressure with application to Lennard-Jones liquid films. J. Colloid Interface Sci. 313 (2), 579-591.

Yonemoto, Y. \& KunUgi, T. 2008 Reconsideration of Young's equation. Thermal Sci. Engng 16 (1), 49-60.

ZhANG, J., WATSON, S. J. \& Wong, H. 2007 Fluid flow and heat transfer in a dual-wet micro heat pipe. J. Fluid Mech. 589, 1-31. 